\title{
Discounted optimal stopping problems for maxima of geometric Brownian motions with switching payoffs
}

\author{
Pavel V. Gapeev* $\quad$ Peter M. Kort ${ }^{\dagger \ddagger} \quad$ Maria N. Lavrutich ${ }^{\S}$
}

\begin{abstract}
We present closed-form solutions to some discounted optimal stopping problems for the running maximum of a geometric Brownian motion with payoffs switching according to the dynamics of a continuous-time Markov chain with two states. The proof is based on the reduction of the original problems to the equivalent free-boundary problems and the solution of the latter problems by means of the smooth-fit and normal-reflection conditions. We show that the optimal stopping boundaries are determined as the maximal solutions of the associated two-dimensional systems of first-order nonlinear ordinary differential equations. The obtained results are related to the valuation of real switching lookback options with fixed and floating sunk costs in the Black-Merton-Scholes model.
\end{abstract}

\section{Introduction}

The main aim of this paper is to present closed-form solutions to the discounted optimal stopping problems with the values:

$$
V_{i}^{*}=\sup _{\tau} E\left[e^{-r \tau}\left(\left(1-\Theta_{\tau}\right)\left(L_{0} \max _{0 \leq t \leq \tau} X_{t}-K_{0}\right)+\Theta_{\tau}\left(L_{1} \max _{0 \leq t \leq \tau} X_{t}-K_{1}\right)\right)\right]
$$

and

$$
W_{i}^{*}=\sup _{\zeta} E\left[e^{-r \zeta}\left(\left(1-\Theta_{\zeta}\right)\left(L_{0} \max _{0 \leq t \leq \zeta} X_{t}-K_{0} X_{\zeta}\right)+\Theta_{\zeta}\left(L_{1} \max _{0 \leq t \leq \zeta} X_{t}-K_{1} X_{\zeta}\right)\right)\right]
$$

*(Corresponding author) London School of Economics, Department of Mathematics, Houghton Street, London WC2A 2AE, United Kingdom; e-mail: p.v.gapeev@lse.ac.uk

†Tilburg University, CentER, Department of Econometrics and Operations Research, PO Box 90153, 5000 LE Tilburg, The Netherlands; e-mail: kort@uvt.nl

$\ddagger$ University of Antwerp, Department of Economics, Prinsstraat 13, 2000 Antwerp 1, Belgium

$\S$ Norwegian University of Science and Technology, Department of Industrial Economics and Technology Management, 7491 Trondheim, Norway; e-mail: maria.lavrutich@ntnu.no

Mathematics Subject Classification 2010: Primary 60G40, 60G44, 60J65. Secondary 91B25, 60J27, 35R35.

Key words and phrases: Discounted optimal stopping problem, geometric Brownian motion, running maximum process, continuous-time Markov chain, free-boundary problem, instantaneous stopping and smooth fit, normal reflection, perpetual American and real options, a change-of-variable formula with local time on surfaces.

Date: July 28, 2020 
for some given constants $L_{i}>0$ and $K_{i} \geq 0, i=0,1$. Here, for a precise formulation of the problem, we consider a probability space $(\Omega, \mathcal{G}, P)$ with a standard Brownian motion $B=\left(B_{t}\right)_{t \geq 0}$ and a two-state continuous-time Markov chain $\Theta=\left(\Theta_{t}\right)_{t \geq 0}$ with the state space $\{0,1\}$ and transition intensities $\lambda_{i}>0, i=0,1$ (the processes $B$ and $\Theta$ are supposed to be independent under the probability measure $P$ ). We assume that the process $X=\left(X_{t}\right)_{t \geq 0}$ is given by:

$$
X_{t}=x \exp \left(\left(\mu-\sigma^{2} / 2\right) t+\sigma B_{t}\right)
$$

so that it solves the stochastic differential equation:

$$
d X_{t}=\mu X_{t} d t+\sigma X_{t} d B_{t} \quad\left(X_{0}=x\right)
$$

where $x>0$ is fixed, and $\sigma>0, \mu<r$, and $r>0$ are some given constants. For simplicity, we assume that the coefficients of the geometric Brownian motion $X$ are independent of the dynamics of the Markov chain $\Theta$. The consideration of a model for the process $X$ with switching coefficients would lead to less explicit formulas for the values of the optimal stopping problems. In our application, the process $X$ describes the current state of technological progress, which changes over time due to the active process of research and development in a branch of the industry, where $r$ is the discount rate. The running maximum of $X$ can be interpreted as the productivity of the best technology available in the market and corresponds to the best state of technological progress achieved so far under constant returns to scale. Suppose that the suprema in (1.1) and (1.2) are taken over all stopping times $\tau$ and $\zeta$ of the process $(X, \Theta)$, and the expectations there are taken with respect to the risk-neutral probability measure $P$. In this case, the values of (1.1) and (1.2) can be interpreted as the rational (or no-arbitrage) values of (perpetual) real lookback switching options with present values, which are linear in the running maximum of $X$, as well as sunk cost investment amounts, which are constant or linear in $X$, in the Black-Merton-Scholes model, respectively (see, e.g. Dixit and Pindyck [12; pages 303-309] for the examples of standard real options with switching payoffs).

Our specification of productivity development is suitable for the industries, such as green energy production, for which technological progress can be characterised by steady and continuous improvements not essentially influencing the resulting market price, and where the productivity linearly depends on the state of the technology. In addition to the technological uncertainty, the profitability of investment projects in the green energy industry is affected by the uncertainty in the government regulation. In fact, the regulatory mechanisms aimed to support the green energy producers have frequently and unexpectedly been revised. Chronopoulos et al. [9] note that green energy investments rely on government support, but the absence of a clear policy framework increases uncertainty in revenue streams. In our model, the policy is present in the form of a subsidy with the aim to stimulate green energy projects. The subsidy switches between the implementation state when the subsidy is "on" and the abolishment state when the subsidy is "off". In several countries, such as Spain, Belgium, Czechia, Bulgaria and others, the subsidies for green energy producers were retroactively adjusted, which considerably reduced the profitability of the existing plants (see, e.g. Dalby et al. [11]). The idea of having switching payoffs as a result of policy uncertainty originally comes from Hassett and Metcalf [27] (see also [12; pages 303-309]). They argue that government regulation is of a mean-reverting nature and therefore introduce the payoffs switching back and forth as a result of policy uncertainty. Once subsidies are introduced, the debate starts about retracting them and the other way round. In 
addition, this situation can, for instance, be motivated by a change in governments leading to different environmental policies. The implications of policy uncertainty was analysed in other recent contributions like Boomsma et al. [5], Boomsma and Linnerud [4], Adkins and Paxson [1], Eryilmaz and Homans [15], Ritzenhofer and Spinler [42].

For the case of non-switching payoffs with $L_{0}=L_{1}$ and $K_{0}=K_{1}$, the problems of (1.1) and (1.2) were solved by Pedersen [34], Guo and Shepp [24], and Beibel and Lerche [8], for models with geometric Brownian motions, and in [16], for a geometric model driven by a Brownian motion and a compound Poisson process with exponential jumps. More recently, Guo and Zervos [25] and Rodosthenous and Zervos [40] derived solutions for discounted optimal stopping problems related to the pricing of perpetual American options with more general payoff functions depending on the current values of the process $X$ and its running maximum. In the case of a Russian option with $L_{0} \neq L_{1}$ and $K_{i}=0, i=0,1$, the problems of (1.1) and (1.2) were explicitly solved by Guo [23] for a model with geometric Brownian motions with switching coefficients. In contrast to the switching Russian option problem studied in [23], the problem of (1.1) is necessarily three-dimensional in the sense that it cannot be reduced to an optimal stopping problem for a two-dimensional (time-homogeneous strong) Markov process having $\Theta$ as one of its state-space components. It is shown that the optimal stopping boundaries for the process $X$ can be expressed as functions of the current value of its running maximum also depending on the current state of the Markov chain $\Theta$. We obtain closed-form expressions for the associated value functions and characterise the optimal stopping boundaries as the maximal solutions to the resulting two-dimensional systems of first-order nonlinear ordinary differential equations.

In the case of $L_{0}=L_{1}$ and $K_{i}=0, i=0,1$, the problems of (1.1) and (1.2) provide the classical Russian option problem introduced and explicitly solved by Shepp and Shiryaev [43], by means of reducing the initial problem to an optimal stopping problem for a twodimensional (continuous) Markov process and solving the latter problem by using the smoothfit and normal-reflection conditions. It was further observed by Shepp and Shiryaev [44] that change-of-measure arguments allow for the reduction of the Russian option problem to an optimal stopping problem for a one-dimensional diffusion process with reflection. This feature explained the simplicity of the structure of the solution obtained in [43]. Gerber et al. [20] and Mordecki and Moreira [32] obtained closed form solutions to the perpetual Russian option problems for diffusions with negative exponential jumps. Asmussen et al. [2] derived explicit expressions for the prices of perpetual Russian options in the dense class of Lévy processes with phase-type jumps in both directions by reducing the original problems to first passage time problems and solving the latter problems by means of martingale stopping and WienerHopf factorisation. Avram et al. [3] studied exit problems for spectrally negative Lévy processes and applied the results to solving optimal stopping problems associated with perpetual Russian and American put options. More complicated optimal stopping problems and games within the framework of models based on spectrally negative Lévy processes and their running maxima processes were studied by Baurdoux and Kyprianou [6], Ott [33], Kyprianou and Ott [30], and Baurdoux and van Schaik [7] among others. Optimal stopping problems for three-dimensional continuous Markov processes containing the running maximum or minimum as well as the running maximum drawdown or drawup as components were recently studied by Peskir [37][38], Glover et al. [21], and [17]-[19] among others.

Models of financial markets with parameter values switching according to the dynamics 
of continuous-time Markov chains have recently found a considerable amount of applications. For instance, the closed-form solutions to the perpetual American lookback and put option pricing problems were obtained by Guo [23] and Guo and Zhang [26] in an extension of such a diffusion model with both the drift and volatility coefficients of the underlying asset price process switching between two constant values according to the change in the state of the observable continuous-time Markov chain. Jobert and Rogers [29] considered the perpetual American put option problem within an extension of that model to the case of several states for the Markov chain, and solved numerically the corresponding problem with finite expiry. In a model with a two-state Markov chain and no diffusion part, Dalang and Hongler [10] presented a complete and essentially explicit solution to a similar problem, which exhibited a surprisingly rich structure. These results were further extended by Jiang and Pistorius [28], who studied the perpetual American put option problem within the framework of an exponential jump-diffusion model with observable dynamics of regime-switching behaviour parameters.

The rest of the paper is organised as follows. In Section 2, we formulate the fixed sunk costs real switching lookback option optimal stopping problem for a necessarily three-dimensional continuous-time Markov process which has the current states of the technological progress $X$ and its running maximum, as well as the Markov chain $\Theta$, as the state-space components. The original optimal stopping problem is reduced to the associated free-boundary problem for the value function which satisfies the smooth-fit conditions at the optimal stopping boundaries and the normal-reflection conditions at the edges of the state space of the three-dimensional process. In Section 3, we obtain closed-form solutions of the associated free-boundary problem and derive systems of two first-order nonlinear ordinary differential equations for the sought boundaries as well as specify their asymptotic behaviour under various relations between the parameters of the model. In Section 4, we apply the change-of-variable formula with local time on surfaces from Peskir [36], to verify that the resulting solution of the free-boundary problem provides the expressions for the value function and the optimal stopping boundaries for the current state of the technological progress in the original problem. In Section 5, we present explicit solutions of the associated floating sunk costs real switching lookback option optimal stopping problem under various relations between the parameters of the model. The main results of the paper are stated in Theorems 4.1 and 5.1 .

\section{Preliminaries}

In this section, we describe the structure of the three-dimensional optimal stopping problem of (1.1) which is related to the fixed sunk costs real switching lookback option pricing problem. For the problem of (1.1), we formulate an equivalent free-boundary problem.

2.1 The optimal stopping problem. It is seen that the problem of (1.1) can be embedded to the optimal stopping problem for the (time-homogeneous strong) Markov process $(X, S, \Theta)=$ $\left(X_{t}, S_{t}, \Theta_{t}\right)_{t \geq 0}$ with the value function:

$$
V_{i}^{*}(x, s)=\sup _{\tau} E_{x, s, i}\left[e^{-r \tau}\left(\left(1-\Theta_{\tau}\right)\left(L_{0} S_{\tau}-K_{0}\right)+\Theta_{\tau}\left(L_{1} S_{\tau}-K_{1}\right)\right)\right]
$$

for some $L_{i}>0$ and $K_{i} \geq 0, i=0,1$, fixed, where the supremum is taken over all stopping times $\tau$ with respect to the natural filtration $\left(\mathcal{G}_{t}\right)_{t \geq 0}$ of the process $(X, \Theta)$. Here, $S=\left(S_{t}\right)_{t \geq 0}$ 
is the associated with $X$ running maximum process defined by:

$$
S_{t}=s \vee\left(\max _{0 \leq u \leq t} X_{u}\right)
$$

for $s \geq x>0$. We denote by $E_{x, s, i}$ the expectation with respect to the probability measure $P_{x, s, i}$ under which the three-dimensional (strong Markov) process $(X, S, \Theta)$ starts at $(x, s, i) \in$ $E \times\{0,1\}$, and by $E=\left\{(x, s) \in \mathbb{R}^{2} \mid 0<x \leq s\right\}$ the state space of the process $(X, S)$. We further assume that $\Theta$ has the transition-probability matrix $\left\{\left(\lambda_{0} e^{-\left(\lambda_{0}+\lambda_{1}\right) t}+\lambda_{1}\right) /\left(\lambda_{0}+\right.\right.$ $\left.\left.\lambda_{1}\right), \lambda_{0}\left(1-e^{-\left(\lambda_{0}+\lambda_{1}\right) t}\right) /\left(\lambda_{0}+\lambda_{1}\right) ; \lambda_{1}\left(1-e^{-\left(\lambda_{0}+\lambda_{1}\right) t}\right) /\left(\lambda_{0}+\lambda_{1}\right),\left(\lambda_{1} e^{-\left(\lambda_{0}+\lambda_{1}\right) t}+\lambda_{0}\right) /\left(\lambda_{0}+\lambda_{1}\right)\right\}$, and the intensity-matrix $\left\{-\lambda_{0}, \lambda_{0} ; \lambda_{1},-\lambda_{1}\right\}$, for all $t \geq 0$, and some $\lambda_{i}>0, i=0,1$, fixed. In other words, the Markov chain $\Theta$ called a telegraphic signal process changes its state from $i$ to $1-i$ at exponentially distributed times of intensity $\lambda_{i}, i=0,1$ (see, e.g. [31; Chapter IX, Section 4] or [14; Chapter VIII]). It can be assumed without loss of generality that $\lambda_{i}$ is the government subsidy implementation intensity and $\lambda_{1-i}$ is the abolishment intensity, for any $i=0,1$ fixed.

2.2 Structure of the optimal stopping time. Let us first determine the structure of the optimal stopping time in the problem of (2.1).

(i) By applying the arguments from [13; Subsection 3.2] and [35; Proposition 2.1] to the optimal stopping problem in (2.1), we see that it is never optimal to stop when $X_{t}=S_{t}$, for each $t \geq 0$ (this fact will be also reproved independently in part (iii) below). It also follows directly from the structure of (2.1) that it is never optimal to stop when $S_{t} \leq K_{i} / L_{i}$, for all $t \geq 0$ and any $i=0,1$ fixed. In other words, this fact shows that all points $(x, s)$ from the set:

$$
C_{i}^{\prime}=\left\{(x, s) \in E \mid 0<x \leq s \leq K_{i} / L_{i}\right\}
$$

and from the diagonal $\left\{(x, s) \in \mathbb{R}^{2} \mid 0<x=s\right\}$ belong to the continuation region:

$$
C_{i}^{*}=\left\{(x, s) \in E \mid V_{i}^{*}(x, s)>L_{i} s-K_{i}\right\}
$$

for every $i=0,1$. It is seen from the solution below that $V_{i}^{*}(x, s)$ is continuous, so that $C_{i}^{*}$ is open, for $i=0,1$.

(ii) It follows from the definition of the process $(X, S)$ in (1.3)-(1.4) and (2.2) and the structure of the reward in (2.1) that, for each $s>K_{i} / L_{i}$ fixed, there exists a sufficiently small $x>0$ such that the point $(x, s)$ belongs to the stopping region:

$$
D_{i}^{*}=\left\{(x, s) \in E \mid V_{i}^{*}(x, s)=L_{i} s-K_{i}\right\}
$$

for every $i=0,1$. This property can be explained by the fact that the costs of waiting until the process $X$ coming from such a small $x>0$ increases to the current value of the running maximum process $S$ may be too large due to the presence of the discounting factor in the reward functional in (2.1). Let us now denote by $\tau^{*}=\tau^{*}(x, s, i)$ the optimal stopping time in the problem of $(2.1)$ and take another starting point $\left(x^{\prime}, s, i\right)$ for the process $(X, S, \Theta)$ such that $0<x^{\prime}<x<s$ and $s>K_{i} / L_{i}$, for any $i=0,1$ fixed. Then, using the fact that the running maximum $S$ from (2.2) of the process $X$ from (1.3) started at the point $x^{\prime}$ is less or equal to the running maximum started at $x$, by virtue of the linear structure of the reward functional in (2.1), we get $V_{i}^{*}\left(x^{\prime}, s\right) \leq V_{i}^{*}(x, s)=L_{i} s-K_{i}$, so that $\left(x^{\prime}, s\right) \in D_{i}^{*}$, for $i=0,1$. On the 
other hand, we can take a point $(x, s) \in C_{i}^{*}$ and consider the associated optimal stopping time $\tau^{*}=\tau^{*}(x, s, i)$, for every $i=0,1$. Hence, for any other starting point $\left(x, s^{\prime}, i\right)$ of the process $(X, S, \Theta)$ such that $0<K_{i} / L_{i} \leq x \leq s^{\prime}<s$, we obtain:

$$
\begin{aligned}
& V_{i}^{*}\left(x, s^{\prime}\right)-\left(L_{i} s^{\prime}-K_{i}\right) \\
& \geq E_{x, s^{\prime}, i}\left[e^{-r \tau^{*}}\left(\left(1-\Theta_{\tau^{*}}\right)\left(L_{0} S_{\tau^{*}}-K_{0}\right)+\Theta_{\tau^{*}}\left(L_{1} S_{\tau^{*}}-K_{1}\right)\right)\right]-\left(L_{i} s^{\prime}-K_{i}\right) \\
& \geq E_{x, s, i}\left[e^{-r \tau^{*}}\left(\left(1-\Theta_{\tau^{*}}\right)\left(L_{0} S_{\tau^{*}}-K_{0}\right)+\Theta_{\tau^{*}}\left(L_{1} S_{\tau^{*}}-K_{1}\right)\right)\right]-\left(L_{i} s-K_{i}\right) \\
& =V_{i}^{*}(x, s)-\left(L_{i} s-K_{i}\right)>0
\end{aligned}
$$

so that $\left(x, s^{\prime}\right) \in C_{i}^{*}$, for $i=0,1$. Thus, combining these arguments together with the comments in [13; Subsection 3.3] and [35; Subsection 3.3], we may therefore conclude that there exists a non-decreasing function $g_{i}^{*}(s)$, for $s>K_{i} / L_{i}$ and $i=0,1$, such that the continuation region $C_{i}^{*}$ in (2.4) is an open set consisting of $C_{i}^{\prime}$ from (2.3) and of the set:

$$
C_{i}^{*} \backslash C_{i}^{\prime}=\left\{(x, s) \in E \mid g_{i}^{*}(s)<x \leq s, s>K_{i} / L_{i}\right\}
$$

while the stopping region $D_{i}^{*}$ in $(2.5)$ is a closed set of the form:

$$
D_{i}^{*}=\left\{(x, s) \in E \mid 0<x \leq g_{i}^{*}(s), s \geq K_{i} / L_{i}\right\}
$$

for $i=0,1$. Note that the existence of such a boundary $g_{i}^{*}(s)$ can also be deduced from the convexity of the function $x \mapsto V_{i}^{*}(x, s)$ on $(0, s)$, for each $s>K_{i} / L_{i}$ and every $i=0,1$.

(iii) Let us now determine the location of the optimal stopping boundaries $g_{i}^{*}(s), i=0,1$. For this purpose, we denote by $U_{i}^{*}(x, s)$ the value function of the optimal stopping problem in (5.1) below, which can be obtained from the one of (2.1), by means of setting $K_{i}=0$, so that $U_{i}^{*}(x, s)=W_{i}^{*}(x, s ; 0,0)$ with $W_{i}^{*}(x, s) \equiv W_{i}^{*}\left(x, s ; K_{0}, K_{1}\right)$ defined in (5.1), for every $i=0,1$. The resulting problem reflects the extreme case scenario in which the change of the policy affects only the revenue of the firm, while the costs always remain constant at a level of zero. It is shown in [23] that the function $U_{i}^{*}(x, s)=W_{i}^{*}(x, s ; 0,0)$ with $W_{i}^{*}(x, s) \equiv$ $W_{i}^{*}\left(x, s ; K_{0}, K_{1}\right)$ from (5.1) admits the explicit expressions in (5.29) and (5.30) below, and the optimal stopping time has the structure $\eta^{*}=\inf \left\{t \geq 0 \mid X_{t} \leq a_{\Theta_{t}}^{*} S_{t}\right\}$, where $a_{i}^{*}$ is defined by $a_{i}^{*}=b_{i}^{*}(0,0)$ with $b_{i}^{*} \equiv b_{i}^{*}\left(K_{0}, K_{1}\right)$ determined from the expressions in (5.15) with (5.16), or (5.19) and (5.22), or (5.25) and (5.28), for every $i=0,1$ (see Theorem 5.1 below). Suppose that $a_{i}^{*} s<g_{i}^{*}(s)$ holds, for some $s>K_{i} / L_{i}, i=0,1$. Then, for each $x \in\left(a_{i}^{*} s, g_{i}^{*}(s)\right)$ given and fixed, we would have $U_{i}^{*}(x, s)-K_{i}>L_{i} s-K_{i}=V_{i}^{*}(x, s)$, contradicting the obvious fact that $U_{i}^{*}(x, s)-K_{i} \leq V_{i}^{*}(x, s)$, for all $(x, s) \in E$ with $s>K_{i} / L_{i}, i=0,1$, as it is clearly seen from the structure of the payoff in (2.1). Thus, we may conclude that $g_{i}^{*}(s) \leq a_{i}^{*} s<s$ should hold, for all $s>K_{i} / L_{i}$ and every $i=0,1$ (see Figure 1 below for a computer drawing of the optimal stopping boundaries $\left.g_{i}^{*}(s), i=0,1\right)$.

(iv) In order to further characterise the asymptotic behaviour of the boundaries $g_{i}^{*}(s)$, $i=0,1$, we observe that for the value functions $V_{i}^{*}(x, s)$ from $(2.1)$ and $U_{i}^{*}(x, s)=W_{i}^{*}(x, s ; 0,0)$ with $W_{i}^{*}(x, s) \equiv W_{i}^{*}\left(x, s ; K_{0}, K_{1}\right)$ from (5.1) the inequalities:

$$
0 \leq U_{i}^{*}(x, s)-K_{i} \leq V_{i}^{*}(x, s) \leq U_{i}^{*}(x, s)
$$


are satisfied, for all $(x, s) \in E$ such that $s>K_{i} / L_{i}, i=0,1$, so that the inequalities:

$$
0 \leq \frac{U_{i}^{*}(s, s)}{s}-\frac{K_{i}}{s} \leq \frac{V_{i}^{*}(s, s)}{s} \leq \frac{U_{i}^{*}(s, s)}{s}
$$

hold, for all $s>K_{i} / L_{i}$ and every $i=0,1$. Therefore, letting $s$ go to infinity in (2.10), we obtain:

$$
\liminf _{s \rightarrow \infty} \frac{V_{i}^{*}(s, s)}{s}=\limsup _{s \rightarrow \infty} \frac{V_{i}^{*}(s, s)}{s}=\lim _{s \rightarrow \infty} \frac{U_{i}^{*}(s, s)}{s}
$$

for $i=0,1$.

2.3 The free-boundary problem. By means of standard arguments based on an application of Itô's formula, it is shown that the infinitesimal operator $\mathbb{L}$ of the process $(X, S)$ from (1.3)-(1.4) and (2.2) acts on an arbitrary function $F(x, s)$ from the class $C^{2,1}$ on $E$ according to the rule:

$$
(\mathbb{L} F)(x, s)=\mu x \partial_{x} F(x, s)+\frac{\sigma^{2} x^{2}}{2} \partial_{x x} F(x, s)
$$

for all $0<x<s$. In order to find analytic expressions for the unknown value functions $V_{i}^{*}(x, s)$ from (2.1) and the unknown boundaries $g_{i}^{*}(s), i=0,1$, from (2.7)-(2.8), let us use the results of general theory of optimal stopping problems for Markov processes (see, e.g. [39; Chapter IV, Section 8]) as well as optimal stopping problems for maximum processes (see, e.g. [39; Chapter V, Sections 15-20] and references therein). We can therefore reduce the optimal stopping problem of (2.1) to the equivalent free-boundary problem:

$$
\begin{aligned}
& \left(\mathbb{L} V_{i}-\left(r+\lambda_{i}\right) V_{i}\right)(x, s)=-\lambda_{i} V_{1-i}(x, s) \quad \text { for } \quad(x, s) \in C_{i} \quad \text { such that } x \neq s \\
& \left.V_{i}(x, s)\right|_{x=g_{i}(s)+}=L_{i} s-K_{i} \quad(\text { instantaneous stopping }) \\
& \left.\partial_{x} V_{i}(x, s)\right|_{x=g_{i}(s)+}=0 \quad(\text { smooth fit }) \\
& \left.\partial_{s} V_{i}(x, s)\right|_{x=s-}=0 \quad(\text { normal reflection }) \\
& V_{i}(x, s)=L_{i} s-K_{i} \quad \text { for } \quad(x, s) \in D_{i} \\
& V_{i}(x, s)>L_{i} s-K_{i} \quad \text { for } \quad(x, s) \in C_{i} \\
& \left(\mathbb{L} V_{i}-\left(r+\lambda_{i}\right) V_{i}\right)(x, s)<-\lambda_{i} V_{1-i}(x, s) \quad \text { for } \quad(x, s) \in D_{i}
\end{aligned}
$$

where $C_{i}$ and $D_{i}$ are defined as $C_{i}^{*}$ and $D_{i}^{*}$ in (2.3) and (2.7)-(2.8) with $g_{i}(s)$ instead of $g_{i}^{*}(s)$, $i=0,1$, respectively. Observe that the superharmonic characterisation of the value function (see, e.g. [39; Chapter IV, Section 9]) implies that $V_{i}^{*}(x, s)$ are the smallest function satisfying (2.13)-(2.14) and (2.17)-(2.18) with the boundaries $g_{i}^{*}(s), i=0,1$. Here, the condition of (2.14) is satisfied, for all $s \geq K_{i} / L_{i}$, while the conditions of (2.15)-(2.16) are satisfied, for all $s>K_{i} / L_{i}$ and any $i=0,1$ fixed.

\section{Solution to the free-boundary problem}

In this section, we obtain solutions to the free-boundary problem of (2.13)-(2.19) and derive systems of ordinary differential equations for the candidate optimal stopping boundaries in 
(2.7)-(2.8) under various relations between the parameters of the model in (1.3)-(1.4) and (2.2). For simplicity, we further assume that $K_{i} / L_{i}<K_{1-i} / L_{1-i}$ holds, for some $i=0,1$ fixed. This assumption, which is particularly satisfied if $L_{i}>L_{1-i}$ and $K_{i}<K_{1-i}$, also yields that $V_{i}(x, s) \geq V_{1-i}(x, s)$, so that $g_{1-i}(s) \leq g_{i}(s)$ holds, for all $s \geq K_{1-i} / L_{1-i}$ and any $i=0,1$ fixed. In this case, the subsidy may positively influence the investment payoff, for instance, by a feed-in premium implying that $L_{i}>L_{1-i}$, or by an investment grant, so that $K_{i}<K_{1-i}$, for $i=0,1$. The cases corresponding to the other relations between the costs rates $L_{i}$ and $K_{i}$, $i=0,1$, can be considered in a similar way.

3.1 The solution to the problem in the case of $\lambda_{i}>0, i=0,1$. Let us first assume that $\lambda_{i}>0, i=0,1$, holds. In this case, following the arguments of [12; pages 303-309], we introduce the functions $\bar{V}_{0}(x, s)=V_{0}(x, s) / \lambda_{0}+V_{1}(x, s) / \lambda_{1}$ and $\bar{V}_{1}(x, s)=V_{1}(x, s)-V_{0}(x, s)$, for all $0<x \leq s$.

(i) It is shown by means of straightforward calculations that the functions $\bar{V}_{j}(x, s), j=0,1$, satisfy the system of second-order ordinary differential equations:

$$
\mu x \partial_{x} \bar{V}_{j}(x, s)+\frac{\sigma^{2} x^{2}}{2} \partial_{x x} \bar{V}_{j}(x, s)-\left(r+\left(\lambda_{0}+\lambda_{1}\right) j\right) \bar{V}_{j}(x, s)=0
$$

for all $g_{i}(s)<x<s$ and $s>K_{1-i} / L_{1-i}$, and any $i=0,1$ fixed. It follows that the equations in (3.1) admit the general solutions:

$$
\bar{V}_{j}(x, s)=\bar{C}_{j, 1}(s) x^{\gamma_{j, 1}}+\bar{C}_{j, 2}(s) x^{\gamma_{j, 2}}
$$

for $g_{i}(s)<x<s$ and $s>K_{1-i} / L_{1-i}$, where $\bar{C}_{j, k}(s)$ are some arbitrary (continuously differentiable) functions, and $\gamma_{j, k}, k=1,2$, are explicitly given by:

$$
\gamma_{j, k}=\frac{1}{2}-\frac{\mu}{\sigma^{2}}-(-1)^{k} \sqrt{\left(\frac{1}{2}-\frac{\mu}{\sigma^{2}}\right)^{2}+\frac{2\left(r+\left(\lambda_{0}+\lambda_{1}\right) j\right)}{\sigma^{2}}}
$$

so that $\gamma_{j, 2}<0<1<\gamma_{j, 1}$ holds, for $j=0,1$. Then, the general solution for the system of second-order ordinary differential equations in (2.13) with (2.12) has the form:

$$
V_{j}(x, s)=\sum_{k=1}^{2}\left(\lambda_{j} C_{j, k}(s) x^{\gamma_{j, k}}-(-1)^{j} C_{1-j, k}(s) x^{\gamma_{1-j, k}}\right)
$$

for all $g_{i}(s)<x<s$ and $s>K_{1-i} / L_{1-i}$, where $C_{j, k}(s)$ are some arbitrary (continuously differentiable) functions, for every $j=0,1$ and $k=1,2$. By virtue of the fact that $V_{i}(x, s)=$ $L_{i} s-K_{i}$ for $g_{1-i}(s)<x \leq g_{i}(s)$, it is seen that the general solution of the equation in (2.13) with $(2.12)$ for $V_{1-i}(x, s)$ takes the form:

$$
V_{1-i}(x, s)=D_{1-i, 1}(s) x^{\beta_{1-i, 1}}+D_{1-i, 2}(s) x^{\beta_{1-i, 2}}+\lambda_{1-i}\left(L_{i} s-K_{i}\right) /\left(r+\lambda_{1-i}\right)
$$

for $g_{1-i}(s)<x<g_{i}(s)$ and $s>K_{1-i} / L_{1-i}$, where $D_{1-i, k}(s)$ are some arbitrary (continuously differentiable) functions, and $\beta_{1-i, k}, k=1,2$, are explicitly given by:

$$
\beta_{1-i, k}=\frac{1}{2}-\frac{\mu}{\sigma^{2}}-(-1)^{k} \sqrt{\left(\frac{1}{2}-\frac{\mu}{\sigma^{2}}\right)^{2}+\frac{2\left(r+\lambda_{1-i}\right)}{\sigma^{2}}}
$$




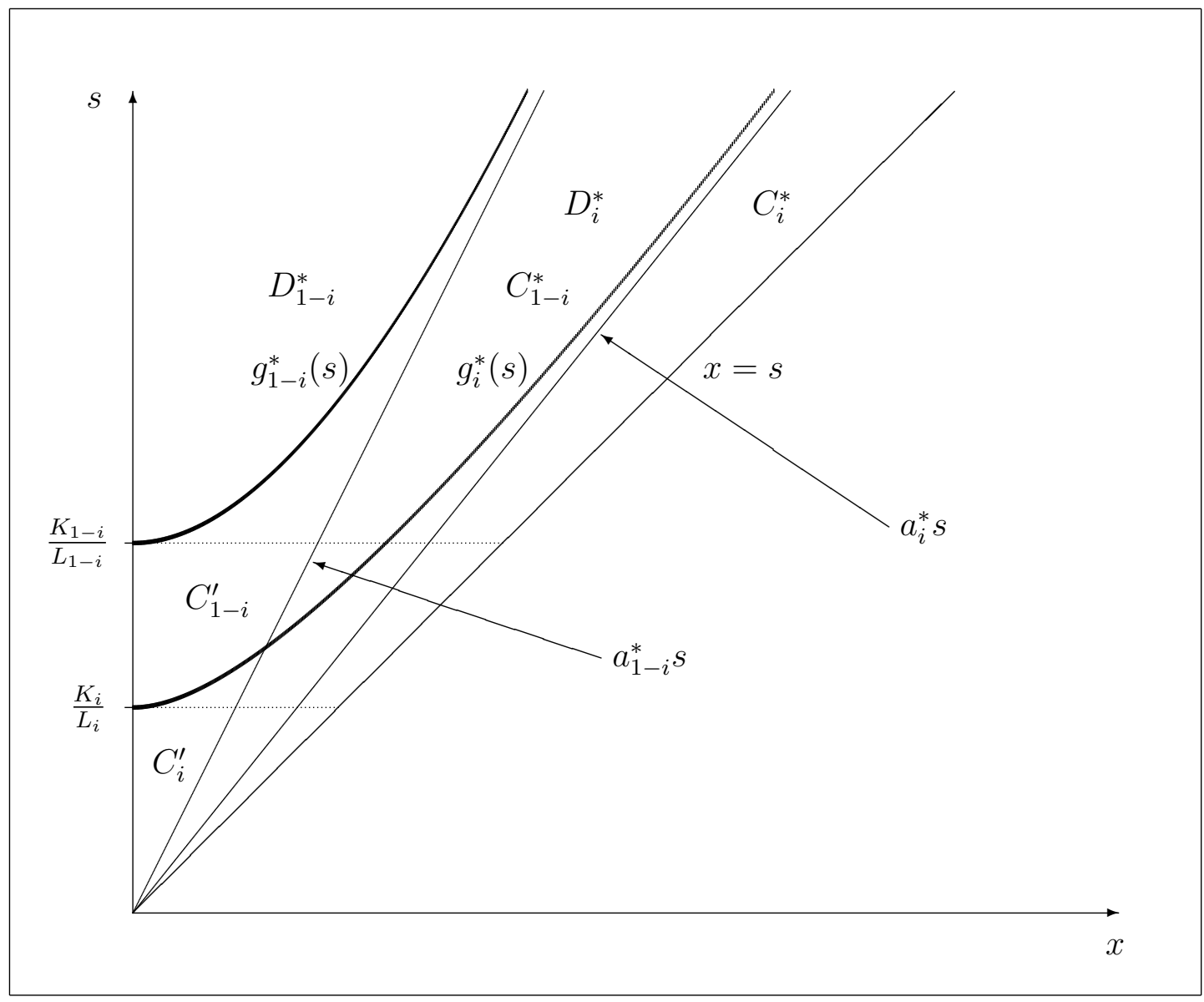

Figure 1. A computer drawing of the optimal stopping boundaries $g_{j}^{*}(s), j=0,1$.

so that $\beta_{1-i, 2}<0<1<\beta_{1-i, 1}$ holds, for $i=0,1$. Hence, by applying the conditions of (2.14)-(2.15) to the functions in (3.4) and (3.5), we obtain that the equalities:

$$
\begin{aligned}
& \sum_{k=1}^{2}\left(\lambda_{i} C_{i, k}(s) g_{i}^{\gamma_{i, k}}(s)-(-1)^{i} C_{1-i, k}(s) g_{i}^{\gamma_{1-i, k}}(s)\right)=L_{i} s-K_{i} \\
& \sum_{k=1}^{2}\left(\lambda_{i} C_{i, k}(s) \gamma_{i, k} g_{i}^{\gamma_{i, k}}(s)-(-1)^{i} C_{1-i, k}(s) \gamma_{1-i, k} g_{i}^{\gamma_{1-i, k}}(s)\right)=0 \\
& D_{1-i, 1}(s) g_{1-i}^{\beta_{1-i, 1}}(s)+D_{1-i, 2}(s) g_{1-i}^{\beta_{1-i, 2}}(s)+\lambda_{1-i}\left(L_{i} s-K_{i}\right) /\left(r+\lambda_{1-i}\right)=L_{1-i} s-K_{1-i} \\
& D_{1-i, 1}(s) \beta_{1-i, 1} g_{1-i}^{\beta_{1-i, 1}}(s)+D_{1-i, 2}(s) \beta_{1-i, 2} g_{1-i}^{\beta_{1-i, 2}}(s)=0
\end{aligned}
$$

hold, for $s>K_{1-i} / L_{1-i}$ and any $i=0,1$ fixed. Moreover, since the function in (3.5) should be 
(at least) twice continuously differentiable at the boundary $g_{i}(s)$, we get that the equalities:

$$
\begin{aligned}
& \sum_{k=1}^{2}\left(\lambda_{1-i} C_{1-i, k}(s) g_{i}^{\gamma_{1-i, k}}(s)-(-1)^{1-i} C_{i, k}(s) g_{i}^{\gamma_{i, k}}(s)\right) \\
& =D_{1-i, 1}(s) g_{i}^{\beta_{1-i, 1}}(s)+D_{1-i, 2}(s) g_{i}^{\beta_{1-i, 2}}(s)+\lambda_{1-i}\left(L_{i} s-K_{i}\right) /\left(r+\lambda_{1-i}\right) \\
& \sum_{k=1}^{2}\left(\lambda_{1-i} C_{1-i, k}(s) \gamma_{1-i, k} g_{i}^{\gamma_{1-i, k}}(s)-(-1)^{1-i} C_{i, k}(s) \gamma_{i, k} g_{i}^{\gamma_{i, k}}(s)\right) \\
& =D_{1-i, 1}(s) \beta_{1-i, 1} g_{i}^{\beta_{1-i, 1}}(s)+D_{1-i, 2}(s) \beta_{1-i, 2} g_{i}^{\beta_{1-i, 2}}(s) \\
& \sum_{k=1}^{2}\left(\lambda_{1-i} C_{1-i, k}(s) \gamma_{1-i, k}\left(\gamma_{1-i, k}-1\right) g_{i}^{\gamma_{1-i, k}}(s)-(-1)^{1-i} C_{i, k}(s) \gamma_{i, k}\left(\gamma_{i, k}-1\right) g_{i}^{\gamma_{i, k}}(s)\right) \\
& =D_{1-i, 1}(s) \beta_{1-i, 1}\left(\beta_{1-i, 1}-1\right) g_{i}^{\beta_{1-i, 1}}(s)+D_{1-i, 2}(s) \beta_{1-i, 2}\left(\beta_{1-i, 2}-1\right) g_{i}^{\beta_{1-i, 2}}(s)
\end{aligned}
$$

hold, for $s>K_{1-i} / L_{1-i}$. Finally, by applying the condition of (2.16) to the functions in (3.4), we conclude that the equalities:

$$
\sum_{k=1}^{2}\left(\lambda_{j} C_{j, k}^{\prime}(s) s^{\gamma_{j, k}}-(-1)^{j} C_{1-j, k}^{\prime}(s) s^{\gamma_{1-j, k}}\right)=0
$$

hold, for all $s>K_{1-i} / L_{1-i}$ and every $j=0,1$.

Solving the system of equations in (3.9)-(3.10), we obtain that the candidate value function admits the representation:

$$
V_{1-i}\left(x, s ; g_{1-i}(s)\right)=\sum_{k=1}^{2} D_{1-i, k}\left(s ; g_{1-i}(s)\right) x^{\beta_{1-i, k}}+\frac{\lambda_{1-i}\left(L_{i} s-K_{i}\right)}{r+\lambda_{1-i}}
$$

for $g_{1-i}(s)<x \leq g_{i}(s)$ and $s>K_{1-i} / L_{1-i}$, with

$$
D_{1-i, k}\left(s ; g_{1-i}(s)\right)=\beta_{1-i, 3-k}\left(L_{1-i}^{\prime} s-K_{1-i}^{\prime}\right) /\left(\left(\beta_{1-i, 3-k}-\beta_{1-i, k}\right) g_{1-i}^{\beta_{1-i, k}}(s)\right)
$$

for $k=1,2$, where we set $L_{1-i}^{\prime}=L_{1-i}-\lambda_{1-i} L_{i} /\left(r+\lambda_{1-i}\right)$ and $K_{1-i}^{\prime}=K_{1-i}-\lambda_{1-i} K_{i} /\left(r+\lambda_{1-i}\right)$, for any $i=0,1$ fixed. Then, solving the system of equations in (3.7)-(3.8) and (3.11)-(3.12), we obtain that the candidate value functions admit the representations:

$$
\begin{aligned}
& V_{j}\left(x, s ; g_{i}(s), g_{1-i}(s)\right) \\
& =\sum_{k=1}^{2}\left(\lambda_{j} C_{j, k}\left(s ; g_{i}(s), g_{1-i}(s)\right) x^{\gamma_{j, k}}-(-1)^{j} C_{1-j, k}\left(s ; g_{i}(s), g_{1-i}(s)\right) x^{\gamma_{1-j, k}}\right)
\end{aligned}
$$

for $g_{i}(s)<x \leq s$ and $s>K_{1-i} / L_{1-i}$, and every $j=0,1$, where the functions $C_{j, k}\left(s ; g_{i}(s), g_{1-i}(s)\right)$, $j=0,1, k=1,2$, admit the representations:

$$
\begin{aligned}
& C_{i, k}\left(s ; g_{i}(s), g_{1-i}(s)\right)=(-1)^{i} /\left(\left(\lambda_{0} \lambda_{1}+1\right)\left(\gamma_{i, 3-k}-\gamma_{i, k}\right) g_{i}^{\gamma_{i, k}}(s)\right) \\
& \times\left(\sum_{l=1}^{2} D_{1-i, l}\left(s ; g_{1-i}(s)\right)\left(\gamma_{i, 3-k}-\beta_{1-i, l}\right) g_{i}^{\beta_{1-i, l}}(s)+\frac{\lambda_{1-i}\left(1+(-1)^{i}\left(r+\lambda_{1-i}\right)\right) \gamma_{i, 3-k}\left(L_{i} s-K_{i}\right)}{r+\lambda_{1-i}}\right)
\end{aligned}
$$


and

$$
\begin{aligned}
& C_{1-i, k}\left(s ; g_{i}(s), g_{1-i}(s)\right)=\lambda_{i} /\left(\left(\lambda_{0} \lambda_{1}+1\right)\left(\gamma_{1-i, 3-k}-\gamma_{1-i, k}\right) g_{i}^{\gamma_{1-i, k}}(s)\right) \\
& \times\left(\sum_{l=1}^{2} D_{1-i, l}\left(s ; g_{1-i}(s)\right)\left(\gamma_{1-i, 3-k}-\beta_{1-i, l}\right) g_{i}^{\beta_{1-i, l}}(s)+\frac{\left(\lambda_{0} \lambda_{1}-(-1)^{i}\left(r+\lambda_{1-i}\right)\right) \gamma_{1-i, 3-k}\left(L_{i} s-K_{i}\right)}{\lambda_{i}\left(r+\lambda_{1-i}\right)}\right)
\end{aligned}
$$

with $D_{1-i, k}\left(s ; g_{1-i}(s)\right), k=1,2$, from (3.16). Hence, we may conclude that the candidate boundaries $g_{j}(s), j=0,1$, satisfy the coupled system of first-order nonlinear ordinary differential equations in (3.14) with:

$$
\begin{aligned}
& C_{i, k}^{\prime}\left(s ; g_{i}(s), g_{i}^{\prime}(s), g_{1-i}(s), g_{1-i}^{\prime}(s)\right)=-C_{i, k}\left(s ; g_{i}(s), g_{1-i}(s)\right) \gamma_{i, k}\left(g_{i}^{\prime}(s) / g_{i}(s)\right) \\
& +\frac{(-1)^{i}}{\left(\lambda_{0} \lambda_{1}+1\right)\left(\gamma_{i, 3-k}-\gamma_{i, k}\right) g_{i}^{\gamma_{i, k}}(s)}\left(\frac{\left(1+(-1)^{i}\left(r+\lambda_{1-i}\right)\right) \gamma_{i, 3-k} L_{i}}{r+\lambda_{1-i}}\right. \\
& \left.+\sum_{l=1}^{2}\left(D_{1-i, l}^{\prime}\left(s ; g_{1-i}(s), g_{1-i}^{\prime}(s)\right)+D_{1-i, l}\left(s ; g_{1-i}(s)\right) \beta_{1-i, l} \frac{g_{i}^{\prime}(s)}{g_{i}(s)}\right)\left(\gamma_{i, 3-k}-\beta_{1-i, l}\right) g_{i}^{\beta_{1-i, l}}(s)\right)
\end{aligned}
$$

and

$$
\begin{aligned}
& C_{1-i, k}^{\prime}\left(s ; g_{i}(s), g_{i}^{\prime}(s), g_{1-i}(s), g_{1-i}^{\prime}(s)\right)=-C_{1-i, k}\left(s ; g_{i}(s), g_{1-i}(s)\right) \gamma_{1-i, k}\left(g_{i}^{\prime}(s) / g_{i}(s)\right) \\
& +\frac{\lambda_{i}}{\left(\lambda_{0} \lambda_{1}+1\right)\left(\gamma_{1-i, 3-k}-\gamma_{1-i, k}\right) g_{i}^{\gamma_{1-i, k}}(s)}\left(\frac{\left(\lambda_{0} \lambda_{1}-(-1)^{i}\left(r+\lambda_{1-i}\right)\right) \gamma_{1-i, 3-k} L_{i}}{\lambda_{i}\left(r+\lambda_{1-i}\right)}\right. \\
& \left.+\sum_{l=1}^{2}\left(D_{1-i, l}^{\prime}\left(s ; g_{1-i}(s), g_{1-i}^{\prime}(s)\right)+D_{1-i, l}\left(s ; g_{1-i}(s)\right) \beta_{1-i, l} \frac{g_{i}^{\prime}(s)}{g_{i}(s)}\right)\left(\gamma_{1-i, 3-k}-\beta_{1-i, l}\right) g_{i}^{\beta_{1-i, l}}(s)\right)
\end{aligned}
$$

as well as

$$
D_{1-i, k}^{\prime}\left(s ; g_{1-i}(s), g_{1-i}^{\prime}(s)\right)=\frac{\beta_{1-i, 3-k}\left(L_{1-i}^{\prime}-\beta_{1-i, k}\left(L_{1-i}^{\prime} s-K_{1-i}^{\prime}\right)\left(g_{1-i}^{\prime}(s) / g_{1-i}(s)\right)\right)}{\left(\beta_{1-i, 3-k}-\beta_{1-i, k}\right) g_{1-i}^{\beta_{1-i, k}}(s)}
$$

for $s>K_{1-i} / L_{1-i}$, where $D_{1-i, k}\left(s ; g_{1-i}(s)\right)$, and $C_{j, k}\left(s ; g_{i}(s), g_{1-i}(s)\right)$, for $i, j=0,1$ and $k=1,2$, are given by the expressions in (3.16) and (3.18)-(3.19), respectively. It is seen that the system of equations in (3.14) is degenerated in the sense that the candidate boundary $g_{i}(s)$ solves the appropriate single first-order nonlinear ordinary differential equation, where the boundary $g_{1-i}(s)$ can be uniquely expressed in terms of $g_{i}(s)$, by means of the arithmetic power equation in (3.13), for any $i=0,1$ fixed.

In order to characterise the asymptotic behaviour of the candidate boundaries $g_{j}(s), j=$ 0,1 , we observe that the expressions in (3.15) and (3.17) imply the representations:

$$
\frac{V_{1-i}\left(s, s ; g_{1-i}(s)\right)}{s}=\sum_{k=1}^{2} \widetilde{D}_{1-i, k}\left(s ; h_{1-i}(s)\right) h_{1-i}^{-\beta_{1-i, k}}(s)+\frac{\lambda_{1-i}\left(L_{i}-K_{i} / s\right)}{r+\lambda_{1-i}}
$$

and

$$
\begin{aligned}
& V_{i}\left(s, s ; g_{i}(s), g_{1-i}(s)\right) / s \\
& =\sum_{k=1}^{2}\left(\lambda_{i} \widetilde{C}_{i, k}\left(s ; h_{i}(s), h_{1-i}(s)\right) h_{i}^{-\gamma_{i, k}}(s)-(-1)^{i} \widetilde{C}_{1-i, k}\left(s ; h_{i}(s), h_{1-i}(s)\right) h_{i}^{-\gamma_{1-i, k}}(s)\right)
\end{aligned}
$$


with some appropriately chosen $\widetilde{D}_{1-i, k}\left(s ; h_{1-i}(s)\right)$ and $\widetilde{C}_{j, k}\left(s ; h_{i}(s), h_{1-i}(s)\right)$, for $i, j=0,1$ and $k=1,2$, which are of constant signs as $s \rightarrow \infty$, where we set $h_{j}(s)=g_{j}(s) / s$, for all $s>K_{j} / L_{j}$ and every $j=0,1$. Then, letting $s$ go to infinity in (3.23) and (3.24) as well as taking into account the assumption that $h_{j}(s) \leq a_{j}^{*}$, for all $s>K_{j} / L_{j}$, so that $\underline{h}_{j} \leq \bar{h}_{j} \leq a_{j}^{*}<1$, we may conclude from the expressions in (2.11) that $\underline{h}_{j}=\bar{h}_{j}=a_{j}^{*}$ should hold, where we set $\underline{h}_{j}=\liminf _{s \rightarrow \infty} h_{j}(s)$ and $\bar{h}_{j}=\limsup _{s \rightarrow \infty} h_{j}(s)$, for every $j=0,1$. Hence, we obtain that the candidate boundaries $g_{j}(s), j=0,1$, should satisfy the property:

$$
\lim _{s \rightarrow \infty} \frac{g_{j}(s)}{s}=a_{j}^{*}
$$

which gives the initial conditions (at infinity) for the equations in (3.14) with (3.20)-(3.21) and (3.22). We also note that the inequalities in (2.9) applied to the functions in (3.15) and (3.17) yield that the candidate boundary $g_{1-i}(s)$ should satisfy the properties:

$$
g_{1-i}\left(\left(K_{1-i} / L_{1-i}\right)+\right)=0 \quad \text { and } \quad g_{1-i}(s) \sim A_{1-i}\left(L_{1-i} s-K_{1-i}\right)^{1 / \beta_{1-i, 1}} \quad \text { as } \quad s \downarrow K_{1-i} / L_{1-i}(3
$$

with some constant $A_{1-i}>0$ which is also specified by means of the condition of (3.25) above.

We further consider the maximal admissible solutions $\left(g_{i}^{*}(s), g_{1-i}^{*}(s)\right)$ of the system of firstorder nonlinear ordinary differential equations in (3.14) with (3.20)-(3.21) and (3.22) as the largest possible solutions which satisfy the inequalities $0<g_{j}^{*}(s) \leq a_{j}^{*} s, j=0,1$, for all $s>K_{1-i} / L_{1-i}$ and every $i=0,1$. By virtue of the classical results on the existence and uniqueness of solutions for systems of first-order nonlinear ordinary differential equations, we may conclude that the system of equations in (3.14) with (3.20)-(3.21) and (3.22) admits (locally) unique solutions, in view of the facts that the Jacobian matrix associated with this implicit system is non-singular and the right-hand sides in the resulting normal (canonic) form correponding to this system are (locally) continuous in $\left(s, g_{i}(s), g_{1-i}(s)\right)$ and (locally) Lipschitz in $g_{i}(s)$ and $g_{1-i}(s)$, for each $s>K_{1-i} / L_{1-i}$ fixed, and every $i=0,1$ (see, e.g. [35; Subsection 3.9]). Then, it is shown by means of technical arguments based on Picard's method of successive approximations that there exists a unique solution $\left(g_{i}(s), g_{1-i}(s)\right)$ to the system of equations in (3.14) with (3.20)-(3.21) and (3.22), for $s>K_{1-i} / L_{1-i}$, started at some point $\left(s_{i, 0}, a_{i}^{*} s_{i, 0}, a_{1-i}^{*} s_{i, 0}\right)$ such that $s_{i, 0}>K_{1-i} / L_{1-i}$, for any $i=0,1$ fixed (see also [22; Subsection 3.2] and [35; Example 4.4] for similar arguments based on the analysis of other single first-order nonlinear ordinary differential equations). Hence, in order to construct the appropriate functions $g_{j}^{*}(s), j=0,1$, which satisfy the system of equations in (3.14) with (3.20)-(3.21) and (3.22) and stay strictly below the lines $a_{j}^{*} s, j=0,1$, we can follow the arguments from [38; Subsection 3.5] (among others) which are based on the construction of sequences of the so-called bad-good solutions which intersect those lines. For this purpose, for any sequences $\left(s_{j, l}\right)_{l \in \mathbb{N}}$ such that $s_{j, l}>K_{1-i} / L_{1-i}$ and $s_{j, l} \uparrow \infty$ as $l \rightarrow \infty$, for every $j=0,1$, we can construct the sequence of solutions $\left(g_{i, l}(s), g_{1-i, l}(s)\right), l \in \mathbb{N}$, to the system in (3.14) with (3.20)-(3.21) and $(3.22)$, for all $s>K_{1-i} / L_{1-i}$ and any $i=0,1$, such that $g_{j, l}\left(s_{j, l}\right)=a_{j}^{*} s_{j, l}$ holds, for each $l \in \mathbb{N}$ and every $j=0,1$. It follows from the form of the arithmetic equations for $a_{i}^{*}=b_{i}^{*}(0,0)$ with $b_{i}^{*} \equiv b_{i}^{*}\left(K_{0}, K_{1}\right)$ in (5.15) with (5.13)-(5.14) and (5.16) below that, for each such solution $\left(g_{i, l}(s), g_{1-i, l}(s)\right)$, the inequality $g_{j, l}^{\prime}\left(s_{j, l}\right)>a_{j}^{*}$ should hold, for each $l \in \mathbb{N}$ and every $i, j=0,1$ (see also [34; pages 979-982] for the analysis of solutions of the single first-order nonlinear differential equation corresponding to the case $L_{0}=L_{1}$ and $\left.K_{0}=K_{1}\right)$. Observe that, by virtue 
of the uniqueness of solutions mentioned above, we know that each two curves $s \mapsto g_{j, l}(s)$ and $s \mapsto g_{j, m}(s)$ cannot intersect, for $l, m \in \mathbb{N}, l \neq m$, and thus, we see that the sequence $\left(g_{j, l}(s)\right)_{l \in \mathbb{N}}$ is decreasing, so that the $\operatorname{limit} g_{j}^{*}(s)=\lim _{l \rightarrow \infty} g_{j, l}(s)$ exists, for each $s>K_{1-i} / L_{1-i}$ and every $i, j=0,1$ fixed. We may therefore conclude that $\left(g_{i}^{*}(s), g_{1-i}^{*}(s)\right)$ provides the maximal solution to the system of equations in (3.14) with (3.20)-(3.21) and (3.22) such that $0<g_{j}^{*}(s) \leq a_{j}^{*} s$ holds, for all $s>K_{1-i} / L_{1-i}$ and every $i, j=0,1$. Moreover, since the right-hand sides of the corresponding normal form of the system of first-order nonlinear ordinary differential equations in (3.14) with (3.20)-(3.21) and (3.22) are (locally) Lipschitz in $s$, for $s>K_{1-i} / L_{1-i}$ and every $i=0,1$, one can deduce by means of Gronwall's inequality that the functions $g_{j, l}(s), l \in \mathbb{N}$, are continuous, so that the functions $g_{j}^{*}(s), j=0,1$, are continuous too. The corresponding maximal admissible solutions of first-order nonlinear ordinary differential equations and the associated maximality principle for solutions of optimal stopping problems which is equivalent to the superharmonic characterisation of the payoff functions were established in [35] and further developed in [22], [34], [24], [16], [6], [25], [37]-[38], [21], [33], [30], [7], [17]-[19], and [40] among other subsequent papers (see also [39; Chapter I; Chapter V, Section 17] for other references).

(ii) In order to find the candidate value functions $V_{j}(x, s)$ and the boundaries $g_{j}(s), j=0,1$, in the region $K_{i} / L_{i}<x \leq s \leq K_{1-i} / L_{1-i}$, we recall that the general solution of (2.13) for $V_{j}(x, s), j=0,1$, takes the form of (3.4), for all $g_{i}(s)<x<s$, while the general solution for $V_{1-i}(x, s)$ is given by (3.5), for all $0<x<g_{i}(s)$ and $K_{i} / L_{i}<s \leq K_{1-i} / L_{1-i}$, for any $i=0,1$ fixed. Then, by applying the conditions of (2.14)-(2.16) to the function in (3.4), we obtain that the equalities (3.7)-(3.8) and (3.14) hold, for $K_{i} / L_{i}<s \leq K_{1-i} / L_{1-i}$ under $j=i$. In this case, the candidate value function $V_{1-i}\left(x, s ; g_{i}(s)\right)$ takes the form:

$$
V_{1-i}\left(x, s ; g_{i}(s)\right)=D_{1-i, 1}\left(s ; g_{i}(s)\right) x^{\beta_{1-i, 1}}+\lambda_{1-i}\left(L_{i} s-K_{i}\right) /\left(r+\lambda_{1-i}\right)
$$

for $0<x \leq g_{i}(s)$, where $D_{1-i, 2}\left(s ; g_{i}(s)\right)=0$ should hold, since otherwise $V_{1-i}\left(x, s ; g_{i}(s)\right) \rightarrow$ $\pm \infty$ as $x \downarrow 0$, that must be excluded, by virtue of the fact that the function $V_{1-i}^{*}(x, s)$ in $(2.1)$ is bounded. Moreover, since the function $V_{1-i}(x, s)$ should be twice continuously differentiable at the boundary $g_{i}(s)$, we get that the equalities in (3.11)-(3.13) are satisfied with $D_{1-i, 1}(s)=$ $D_{1-i, 1}\left(s ; g_{i}(s)\right)$ and $D_{1-i, 2}(s)=0$. Hence, solving the system of equations in (3.7)-(3.8) and (3.11)-(3.13) with $D_{1-i, 1}(s)=D_{1-i, 1}\left(s ; g_{i}(s)\right)$ and $D_{1-i, 2}(s)=0$, we obtain that the candidate value function $V_{i}\left(x, s ; g_{i}(s)\right)$ admits the representation of $(3.17)$ under $j=i$, for $g_{i}(s)<$ $x \leq s$ and $K_{i} / L_{i}<s \leq K_{1-i} / L_{1-i}$, where the functions $C_{i, k}\left(s ; g_{i}(s)\right), k=1,2$, admit the representations in (3.18)-(3.19) with $D_{1-i, 1}\left(s ; g_{i}(s)\right)$ given by:

$$
\begin{aligned}
& D_{1-i, 1}\left(s ; g_{i}(s)\right)=\left(\left(L_{i} s-K_{i}\right) / g_{i}^{\beta_{1-i, 1}}(s)\right) \\
& \times\left(\sum _ { k = 1 } ^ { 2 } \left(\frac{\lambda_{0} \lambda_{1} \gamma_{1-i, k}\left(\gamma_{1-i, k}-1\right)}{\left(\lambda_{0} \lambda_{1}+1\right)\left(\gamma_{1-i, 3-k}-\gamma_{1-i, k}\right)} \frac{\left(\lambda_{0} \lambda_{1}-(-1)^{i}\left(r+\lambda_{1-i}\right)\right) \gamma_{1-i, 3-k}}{\lambda_{i}\left(r+\lambda_{1-i}\right)}\right.\right. \\
& \left.\left.\quad+\frac{\gamma_{i, k}\left(\gamma_{i, k}-1\right)}{\left(\lambda_{0} \lambda_{1}+1\right)\left(\gamma_{i, 3-k}-\gamma_{i, k}\right)} \frac{\lambda_{1-i}\left(1+(-1)^{i}\left(r+\lambda_{1-i}\right)\right) \gamma_{i, 3-k}}{r+\lambda_{1-i}}\right)\right) / \\
& \left(\sum_{k=1}^{2}\left(\frac{\lambda_{0} \lambda_{1} \gamma_{1-i, k}\left(\gamma_{1-i, k}-1\right)\left(\beta_{1-i, 1}-\gamma_{1-i, 3-k}\right)}{\left(\lambda_{0} \lambda_{1}+1\right)\left(\gamma_{1-i, 3-k}-\gamma_{1-i, k}\right)}+\frac{\gamma_{i, k}\left(\gamma_{i, k}-1\right)\left(\beta_{1-i, 1}-\gamma_{i, 3-k}\right)}{\left(\lambda_{0} \lambda_{1}+1\right)\left(\gamma_{i, 3-k}-\gamma_{i, k}\right)}\right)-\beta_{1-i, 1}\left(\beta_{1-i, 1}-1\right)\right)
\end{aligned}
$$

and $D_{1-i, 2}\left(s ; g_{i}(s)\right)=0$, for any $i=0,1$ fixed. Therefore, we may conclude that the candidate boundary $g_{i}(s)$ satisfies the ordinary differential equation in (3.14) under $j=i$ with 
$C_{i, k}\left(s ; g_{i}(s)\right), k=1,2$, determined as in (3.20)-(3.21), and

$$
D_{1-i, 1}^{\prime}\left(s ; g_{i}(s)\right)=D_{1-i, 1}\left(s ; g_{i}(s)\right)\left(L_{i} /\left(L_{i} s-K_{i}\right)-\beta_{1-i, 1}\left(g_{i}^{\prime}(s) / g_{i}(s)\right)\right)
$$

as well as $D_{1-i, 2}^{\prime}\left(s ; g_{i}(s)\right)=0$, for $K_{i} / L_{i}<s<K_{1-i} / L_{1-i}$, for any $i=0,1$ fixed. Observe that it also follows from the properties $V_{1-i}\left(\left(K_{1-i} / L_{1-i}\right)-,\left(K_{1-i} / L_{1-i}\right)-\right)=V_{1-i}\left(K_{1-i} / L_{1-i}, K_{1-i} / L_{1-i}\right)$ and $V_{1-i}^{\prime}\left(\left(K_{1-i} / L_{1-i}\right)-,\left(K_{1-i} / L_{1-i}\right)-\right)=V_{1-i}^{\prime}\left(\left(K_{1-i} / L_{1-i}\right)+,\left(K_{1-i} / L_{1-i}\right)+\right)$ for the the candidate boundary should satisfy the condition $g_{i}\left(\left(K_{1-i} / L_{1-i}\right)-\right)=g_{i}\left(\left(K_{1-i} / L_{1-i}\right)+\right)$, for any $i=0,1$ fixed. We also note that, by virtue of the arguments similar to the ones from the end of part (i) above, the candidate boundary $g_{i}(s)$ should satisfy the properties:

$$
g_{i}\left(\left(K_{i} / L_{i}\right)+\right)=0 \quad \text { and } \quad g_{i}(s) \sim A_{i}\left(L_{i} s-K_{i}\right)^{1 / \gamma_{1,1}} \quad \text { as } \quad s \downarrow K_{i} / L_{i}
$$

with some constant $A_{i}>0$ which is specified by means of the initial condition of (3.25) above.

(iii) In order to find the candidate value functions $V_{j}(x, s), j=0,1$, in the region $0<x \leq$ $s \leq K_{i} / L_{i}$, for any $i=0,1$ fixed, we observe that the expression:

$$
V_{j}^{*}(x, s)=E_{x, j}\left[e^{-r \tau_{i}^{\prime}}\right] V_{j}^{*}\left(K_{i} / L_{i}, K_{i} / L_{i}\right)
$$

holds, for the first hitting time:

$$
\tau_{1-i}^{\prime}=\inf \left\{t \geq 0 \mid X_{t} \geq K_{1-i} / L_{1-i}\right\}
$$

where the candidate solutions for $V_{j}^{*}\left(K_{i} / L_{i}, K_{i} / L_{i}\right), j=0,1$, are determined from parts (i)-(ii) above, for any $i=0,1$ fixed. We recall that the general solution of $(2.13)$ for $V_{j}(x, s), j=0,1$, takes the form of (3.4), for all $0<x<s \leq K_{i} / L_{i}$. Note that, in this case, the candidate value functions $V_{j}\left(x, s ; K_{i} / L_{i}\right), j=0,1$, are given by (3.17), for $0<x \leq s \leq K_{i} / L_{i}$, where $C_{j, 2}\left(s ; g_{i}(s), g_{1-i}(s)\right)=0$ should hold, for every $j=0,1$, since otherwise $V_{j}\left(x, s ; K_{i} / L_{i}\right) \rightarrow \pm \infty$ as $x \downarrow 0$, that must be excluded, by virtue of the fact that the functions $V_{j}^{*}(x, s), j=0,1$, in (3.31) are bounded. Then, by applying the boundary conditions $V_{j}\left(\left(K_{i} / L_{i}\right)-,\left(K_{i} / L_{i}\right)-\right)=$ $V_{j}\left(K_{i} / L_{i}, K_{i} / L_{i}\right)$ to the function in (3.4) with $C_{j, 2}(s)=0, j=0,1$, we get that the candidate value function takes the form:

$$
V_{j}\left(x ; K_{i} / L_{i}\right)=\lambda_{j} C_{j, 1}\left(K_{i} / L_{i}\right) x^{\gamma_{j, 1}}-(-1)^{j} C_{1-j, 1}\left(K_{i} / L_{i}\right) x^{\gamma_{1-j, 1}}
$$

for $0<x \leq s \leq K_{i} / L_{i}$, with

$$
C_{j, 1}\left(K_{i} / L_{i}\right)=\frac{\lambda_{1-j} V_{j}\left(K_{i} / L_{i}, K_{i} / L_{i}\right)+(-1)^{j} V_{1-j}\left(K_{i} / L_{i}, K_{i} / L_{i}\right)}{\left(\lambda_{0} \lambda_{1}+1\right)\left(K_{i} / L_{i}\right)^{\gamma_{j, 1}}}
$$

where the function $V_{j}\left(K_{i} / L_{i}, K_{i} / L_{i}\right)=V_{j}\left(\left(K_{i} / L_{i}\right)+,\left(K_{i} / L_{i}\right)+\right), j=0,1$, is determined in parts (i)-(ii) above, for any $i=0,1$ fixed.

3.2 The solution to the problem in the case of $\lambda_{i}>\lambda_{1-i}=0$. Let us now assume that $\lambda_{i}>\lambda_{1-i}=0$ holds, for any $i=0,1$ fixed.

(i) In this case, the general solution of the second-order ordinary differential equation in (2.13) with $(2.12)$ for $V_{1-i}(x, s)$ has the form:

$$
V_{1-i}(x, s)=C_{1-i, 1}(s) x^{\gamma_{0,1}}+C_{1-i, 2}(s) x^{\gamma_{0,2}}
$$


for all $g_{1-i}(s)<x<s$, where $C_{1-i, k}(s), k=1,2$, are some arbitrary functions, and $\gamma_{0,2}<0<$ $1<\gamma_{0,1}$ are defined in (3.3). Then, by applying the conditions of (2.14)-(2.16) to the function in (3.35), we obtain the equalities:

$$
\begin{aligned}
& C_{1-i, 1}(s) g_{1-i}^{\gamma_{0,1}}(s)+C_{1-i, 2}(s) g_{1-i}^{\gamma_{0,2}}(s)=L_{1-i} s-K_{1-i} \\
& C_{1-i, 1}(s) \gamma_{0,1} g_{1-i}^{\gamma_{0,1}}(s)+C_{1-i, 2}(s) \gamma_{0,2} g_{1-i}^{\gamma_{0,2}}(s)=0 \\
& C_{1-i, 1}^{\prime}(s) s^{\gamma_{0,1}}+C_{1-i, 2}^{\prime}(s) s^{\gamma_{0,2}}=0
\end{aligned}
$$

for $s>K_{1-i} / L_{1-i}$ (see [34] and [24]). Hence, solving the system of equations in (3.36)-(3.37), we obtain that the candidate value function admits the representation:

$$
V_{1-i}\left(x, s ; g_{1-i}(s)\right)=C_{1-i, 1}\left(s ; g_{1-i}(s)\right) x^{\gamma_{0,1}}+C_{1-i, 2}\left(s ; g_{1-i}(s)\right) x^{\gamma_{0,2}}
$$

for $g_{1-i}(s)<x \leq s$, with

$$
C_{1-i, k}\left(s ; g_{1-i}(s)\right)=\gamma_{0,3-k}\left(L_{1-i} s-K_{1-i}\right) /\left(\left(\gamma_{0,3-k}-\gamma_{0, k}\right) g_{1-i}^{\gamma_{0, k}}(s)\right)
$$

for $k=1,2$. Thus, by applying the condition of (3.38) to the functions in (3.40), we conclude that the candidate boundary satisfies the ordinary differential equation:

$$
g_{1-i}^{\prime}(s)=\frac{L_{1-i} g_{1-i}(s)}{L_{1-i} s-K_{1-i}} \frac{\gamma_{0,2}\left(s / g_{1-i}(s)\right)^{\gamma_{0,1}}-\gamma_{0,1}\left(s / g_{1-i}(s)\right)^{\gamma_{0,2}}}{\gamma_{0,1} \gamma_{0,2}\left(\left(s / g_{1-i}(s)\right)^{\gamma_{0,1}}-\left(s / g_{1-i}(s)\right)^{\left.\gamma_{0,2}\right)}\right.}
$$

for $s>K_{1-i} / L_{1-i}$ (see [34] and [24]). It is easily seen that the right-hand side of the expression in (3.41) is strictly positive, so that the candidate boundary $g_{1-i}(s)$ is a strictly increasing function on $\left(K_{1-i} / L_{1-i}, \infty\right)$. We also note that the functions in (3.40) do not depend on the boundary $g_{1-i}(s)$ directly, since the Markov chain $\Theta$ cannot leave the state $1-i$ when $\lambda_{1-i}=0$, for any $i=0,1$ fixed.

Substituting the expression for $V_{1-i}\left(x, s ; g_{1-i}(s)\right)$ from (3.39) with (3.40) into the equation of $(2.13)$ for $V_{i}(x, s)$, we obtain that the general solution has the form:

$$
V_{i}(x, s)=C_{i, 1}(s) x^{\beta_{i, 1}}+C_{i, 2}(s) x^{\beta_{i, 2}}+V_{1-i}\left(x, s ; g_{1-i}(s)\right)
$$

for all $g_{i}(s)<x<s$, where $C_{i, k}(s), k=1,2$, are some arbitrary functions, and $\beta_{i, 2}<0<1<$ $\beta_{i, 1}$ are defined as in (3.6). Then, by applying the conditions of (2.14)-(2.16) to the function in (3.42), we obtain the equalities:

$$
\begin{aligned}
& C_{i, 1}(s) g_{i}^{\beta_{i, 1}}(s)+C_{i, 2}(s) g_{i}^{\beta_{i, 2}}(s)+V_{1-i}\left(g_{i}(s), s ; g_{1-i}(s)\right)=L_{i} s-K_{i} \\
& C_{i, 1}(s) \beta_{i, 1} g_{i}^{\beta_{i, 1}}(s)+C_{i, 2}(s) \beta_{i, 2} g_{i}^{\beta_{i, 2}}(s)+g_{i}(s) \partial_{x} V_{1-i}\left(g_{i}(s), s ; g_{1-i}(s)\right)=0 \\
& C_{i, 1}^{\prime}(s) s^{\beta_{i, 1}}+C_{i, 2}^{\prime}(s) s^{\beta_{i, 2}}+\left(\partial_{s}+g_{1-i}^{\prime}(s) \partial_{g_{1-i}}\right) V_{1-i}\left(s, s ; g_{1-i}(s)\right)=0
\end{aligned}
$$

for all $s>K_{1-i} / L_{1-i}$. In this case, solving the system of equations in (3.43)-(3.44), we obtain that the candidate value function admits the representation:

$$
V_{i}\left(x, s ; g_{i}(s), g_{1-i}(s)\right)=\sum_{k=1}^{2} C_{i, k}\left(s ; g_{i}(s), g_{1-i}(s)\right) x^{\beta_{i, k}}+V_{1-i}\left(x, s ; g_{1-i}(s)\right)
$$


for $g_{i}(s)<x \leq s$, with

$$
\begin{aligned}
& C_{i, k}\left(s ; g_{i}(s), g_{1-i}(s)\right) \\
& =\frac{\partial_{x} V_{1-i}\left(g_{i}(s), s ; g_{1-i}(s)\right)-\beta_{i, 3-k}\left(V_{1-i}\left(g_{i}(s), s ; g_{1-i}(s)\right)-L_{i} s+K_{i}\right)}{\left(\beta_{i, 3-k}-\beta_{i, k}\right) g_{i}^{\beta_{i, k}}(s)}
\end{aligned}
$$

for $k=1,2$. Thus, by applying the condition of (3.45) to the functions in (3.47), we conclude that the candidate boundary satisfies the ordinary differential equation:

$$
\begin{aligned}
& g_{i}^{\prime}(s)=-\left(\left(\partial_{s}+g_{1-i}^{\prime}(s) \partial_{g_{1-i}}\right) V_{1-i}\left(s, s ; g_{1-i}(s)\right)\right. \\
& \left.+\left(\partial_{s}+g_{1-i}^{\prime}(s) \partial_{g_{1-i}}\right) C_{i, 1}\left(s ; g_{i}(s), g_{1-i}(s)\right) s^{\beta_{i, 1}}+\left(\partial_{s}+g_{1-i}^{\prime}(s) \partial_{g_{1-i}}\right) C_{i, 2}\left(s ; g_{i}(s), g_{1-i}(s)\right) s^{\beta_{i, 2}}\right) / \\
& \left(\partial_{g_{i}} C_{i, 1}\left(s ; g_{i}(s), g_{1-i}(s)\right) s^{\beta_{i, 1}}+\partial_{g_{i}} C_{i, 2}\left(s ; g_{i}(s), g_{1-i}(s)\right) s^{\beta_{i, 2}}\right)
\end{aligned}
$$

for $s>K_{1-i} / L_{1-i}$ and any $i=0,1$ fixed. It is seen that the system of equations in (3.41) and (3.48) is degenerated in the sense that the candidate boundary $g_{1-i}(s)$ solves the single first-order nonlinear ordinary differential equation in (3.41), while the boundary $g_{i}(s)$ can be expressed in terms of $g_{1-i}(s)$, by means of the first-order nonlinear ordinary differential equation in (3.48), for any $i=0,1$ fixed.

In order to characterise the asymptotic behaviour of the candidate boundaries $g_{j}(s), j=$ 0,1 , we observe that the expressions in (3.39)-(3.40) and (3.46)-(3.47) imply the representations:

$$
V_{1-i}\left(s, s ; g_{1-i}(s)\right) / s=\widetilde{C}_{1-i, 1}\left(s ; h_{1-i}(s)\right) h_{1-i}^{-\gamma_{0,1}}(s)+\widetilde{C}_{1-i, 2}\left(s ; h_{1-i}(s)\right) h_{1-i}^{-\gamma_{0,2}}(s)
$$

and

$$
\begin{aligned}
& V_{i}\left(s, s ; g_{i}(s), g_{1-i}(s)\right) / s \\
& =\sum_{k=1}^{2}\left(\widetilde{C}_{i, k}\left(s ; h_{i}(s), h_{1-i}(s)\right) h_{i}^{-\beta_{i, k}}(s)+\widetilde{C}_{1-i, k}\left(s ; h_{i}(s), h_{1-i}(s)\right) h_{i}^{-\gamma_{0, k}}(s)\right)
\end{aligned}
$$

with some appropriately chosen $\widetilde{C}_{j, k}\left(s ; h_{i}(s), h_{1-i}(s)\right)$, for $i, j=0,1$ and $k=1,2$, which are of constant signs as $s \rightarrow \infty$, where we recall that $h_{j}(s)=g_{j}(s) / s$, for all $s>K_{j} / L_{j}$ and every $j=0,1$. Then, following the arguments from the end of part (i) of Subsection 3.1, we may therefore conclude that the candidate boundary $g_{1-i}(s)$ should satisfy the conditions of (3.25) as well as the properties:

$$
g_{1-i}\left(\left(K_{1-i} / L_{1-i}\right)+\right)=0 \quad \text { and } \quad g_{1-i}(s) \sim A_{1-i}\left(L_{1-i} s-K_{1-i}\right)^{1 / \gamma_{0,1}} \quad \text { as } \quad s \downarrow K_{1-i} / L_{1-i}
$$

with some constant $A_{1-i}>0$ which is also specified by means of the condition of (3.25) above. It also follows from the arguments of the end of part (i) of Subsection 3.1 above that there exist maximal solutions $g_{j}^{*}(s), j=0,1$, of the first-order nonlinear ordinary differential equations in (3.41) and (3.48) such that the inequalities $0<g_{j}^{*}(s) \leq a_{j}^{*} s$ hold, for $j=0,1$.

(ii) In order to find the candidate value functions $V_{j}(x, s), j=0,1$, and the boundary $g_{i}(s)$ in the region $K_{i} / L_{i}<x \leq s \leq K_{1-i} / L_{1-i}$, we recall that the general solution of (2.13) is given by (3.35), for all $0<x<g_{i}(s)$ and any $i=0,1$ fixed. Note that, in this case, the candidate value function $V_{1-i}\left(x, s ; K_{1-i} / L_{1-i}\right)$ takes the form of $(3.39)$, for $0<x \leq g_{i}(s)$, 
where $C_{1-i, 2}\left(s ; K_{1-i} / L_{1-i}\right)=0$ should hold, since otherwise $V_{1-i}\left(x, s ; K_{1-i} / L_{1-i}\right) \rightarrow \pm \infty$ as $x \downarrow 0$, that must be excluded, by virtue of the fact that the function $V_{1-i}^{*}(x, s)$ in $(2.1)$ is bounded. Hence, by applying the boundary condition $V_{1-i}\left(\left(K_{1-i} / L_{1-i}\right)-,\left(K_{1-i} / L_{1-i}\right)-\right)=$ $V_{1-i}\left(K_{1-i} / L_{1-i}, K_{1-i} / L_{1-i}\right)$ to the function in (3.39) with $C_{1-i, 2}\left(s ; K_{1-i} / L_{1-i}\right)=0$, we get that the candidate value function takes the form:

$$
V_{1-i}\left(x ; K_{1-i} / L_{1-i}\right)=\left(L_{1-i} x / K_{1-i}\right)^{\gamma_{0,1}} V_{1-i}\left(K_{1-i} / L_{1-i}, K_{1-i} / L_{1-i}\right)
$$

for $0<x \leq g_{i}(s), i=0,1$, where the candidate solution $V_{1-i}\left(K_{1-i} / L_{1-i}, K_{1-i} / L_{1-i}\right)=$ $V_{1-i}\left(\left(K_{1-i} / L_{1-i}\right)+,\left(K_{1-i} / L_{1-i}\right)+\right)$ is determined in part (i) above, for any $i=0,1$ fixed.

Substituting the expression for $V_{1-i}\left(x ; K_{1-i} / L_{1-i}\right)$ from (3.52) into the equation of (2.13) for $V_{i}(x, s)$, we obtain that its general solution has the form of $(3.35)$, for $g_{i}(s)<x<s$ and any $i=0,1$ fixed. Then, by applying the conditions of (2.14)-(2.16) to the function in (3.35), we obtain the equalities (3.43)-(3.45), with $V_{1-i}\left(x ; K_{1-i} / L_{1-i}\right)$ given by (3.52), for $K_{i} / L_{i}<x \leq s \leq K_{1-i} / L_{1-i}$. In this case, solving the system of equations in (3.43)-(3.44), we conclude that the candidate value function admits the representation:

$$
V_{i}\left(x, s ; g_{i}(s)\right)=C_{i, 1}\left(s ; g_{i}(s)\right) x^{\beta_{i, 1}}+C_{i, 2}\left(s ; g_{i}(s)\right) x^{\beta_{i, 2}}+V_{1-i}\left(x ; K_{1-i} / L_{1-i}\right)
$$

for $g_{i}(s)<x \leq s$, with

$$
C_{i, k}\left(s ; g_{i}(s)\right)=\frac{V_{1-i}^{\prime}\left(g_{i}(s) ; K_{1-i} / L_{1-i}\right)-\beta_{i, 3-k}\left(V_{1-i}\left(g_{i}(s) ; K_{1-i} / L_{1-i}\right)-L_{i} s+K_{i}\right)}{\left(\beta_{i, 3-k}-\beta_{i, k}\right) g_{i}^{\beta_{i, k}}(s)}
$$

for $k=1,2$. Thus, by applying the condition of (3.45) to the functions in (3.54), we conclude that the candidate boundary satisfies the ordinary differential equation:

$$
g_{i}^{\prime}(s)=-\frac{\partial_{s} C_{i, 1}\left(s ; g_{i}(s)\right) s^{\beta_{i, 1}}+\partial_{s} C_{i, 2}\left(s ; g_{i}(s)\right) s^{\beta_{i, 2}}}{\partial_{g_{i}} C_{i, 1}\left(s ; g_{i}(s)\right) s^{\beta_{i, 1}}+\partial_{g_{i}} C_{i, 2}\left(s ; g_{i}(s)\right) s^{\beta_{i, 2}}}
$$

for $K_{i} / L_{i}<s<K_{1-i} / L_{1-i}$. We also note that, by virtue of the arguments similar to the ones from the end of part (i) above, the candidate boundary $g_{i}(s)$ should satisfy the properties:

$$
g_{i}\left(\left(K_{i} / L_{i}\right)+\right)=0 \quad \text { and } \quad g_{i}(s) \sim A_{i}\left(L_{i} s-K_{i}\right)^{1 / \beta_{i, 1}} \quad \text { as } \quad s \downarrow K_{i} / L_{i}
$$

with some constant $A_{i}>0$ which is specified by means of the initial condition of (3.25) above.

(iii) In order to find the candidate value functions $V_{j}(x, s), j=0,1$, in the region $0<$ $x \leq s \leq K_{i} / L_{i}$, we recall that the general solution of (2.13) is given by (3.35), for all $0<$ $x<s \leq K_{i} / L_{i}$ and any $i=0,1$ fixed. Note that, in this case, the candidate value functions $V_{j}\left(x, s ; K_{i} / L_{i}\right)$ take the form of (3.39), for $0<x \leq s \leq K_{i} / L_{i}$, where $C_{j, 2}\left(s ; K_{i} / L_{i}\right)=0$ should hold, for every $j=0,1$, since otherwise $V_{j}\left(x, s ; K_{i} / L_{i}\right) \rightarrow \pm \infty$ as $x \downarrow 0$, that must be excluded, by virtue of the fact that the functions $V_{j}^{*}(x, s), j=0,1$, in (3.31) are bounded. Hence, by applying the boundary condition $V_{j}\left(\left(K_{i} / L_{i}\right)-,\left(K_{i} / L_{i}\right)-\right)=V_{j}\left(K_{i} / L_{i}, K_{i} / L_{i}\right)$ to the function in (3.39) with $C_{j, 2}\left(s ; K_{i} / L_{i}\right)=0, j=0,1$, we get that the candidate value functions take the form:

$$
V_{j}\left(x ; K_{i} / L_{i}\right)=\left(L_{i} x / K_{i}\right)^{\gamma_{0,1}} V_{j}\left(K_{i} / L_{i}, K_{i} / L_{i}\right)
$$

for $0<x \leq s \leq K_{i} / L_{i}$, where the functions $V_{j}\left(K_{i} / L_{i}, K_{i} / L_{i}\right)=V_{j}\left(\left(K_{i} / L_{i}\right)+,\left(K_{i} / L_{i}\right)+\right)$, $j=0,1$, are determined in part (ii) above, for any $i=0,1$ fixed. 
3.3 The solution to the problem in the case of $\lambda_{1-i}>\lambda_{i}=0$. Let us now assume that $\lambda_{1-i}>\lambda_{i}=0$ holds, for any $i=0,1$ fixed.

(i) In this case, the general solution of the second-order ordinary differential equation in (2.13) with $(2.12)$ for $V_{i}(x, s)$ has the form:

$$
V_{i}(x, s)=C_{i, 1}(s) x^{\gamma_{0,1}}+C_{i, 2}(s) x^{\gamma_{0,2}}
$$

for all $g_{i}(s)<x \leq s$, where $C_{i, k}(s), k=1,2$, are some arbitrary functions and $\gamma_{0,2}<0<1<$ $\gamma_{0,1}$ are given by (3.3) above. Then, by applying the conditions of (2.14)-(2.16) to the function in (3.58), we obtain the equalities:

$$
\begin{aligned}
& C_{i, 1}(s) g_{i}^{\gamma_{0,1}}(s)+C_{i, 2}(s) g_{i}^{\gamma_{0,2}}(s)=L_{i} s-K_{i} \\
& C_{i, 1}(s) \gamma_{0,1} g_{i}^{\gamma_{0,1}}(s)+C_{i, 2}(s) \gamma_{0,2} g_{i}^{\gamma_{0,2}}(s)=0 \\
& C_{i, 1}^{\prime}(s) s^{\gamma_{0,1}}+C_{i, 2}^{\prime}(s) s^{\gamma_{0,2}}=0
\end{aligned}
$$

for $s>K_{i} / L_{i}$ (see [34] and [24]). Hence, solving the system of equations in (3.59)-(3.60), we obtain that the candidate value function admits the representation:

$$
V_{i}\left(x, s ; g_{i}(s)\right)=C_{i, 1}\left(s ; g_{i}(s)\right) x^{\gamma_{0,1}}+C_{i, 2}\left(s ; g_{i}(s)\right) x^{\gamma_{0,2}}
$$

for $g_{i}(s)<x \leq s$, with

$$
C_{i, k}\left(s ; g_{i}(s)\right)=\gamma_{0,3-k}\left(L_{i} s-K_{i}\right) /\left(\left(\gamma_{0,3-k}-\gamma_{0, k}\right) g_{i}^{\gamma_{0, k}}(s)\right)
$$

for $k=1,2$. Thus, by applying the condition of (3.61) to the functions in (3.63), we conclude that the candidate boundary satisfies the ordinary differential equation:

$$
g_{i}^{\prime}(s)=\frac{L_{i} g_{i}(s)}{L_{i} s-K_{i}} \frac{\gamma_{0,2}\left(s / g_{i}(s)\right)^{\gamma_{0,1}}-\gamma_{0,1}\left(s / g_{i}(s)\right)^{\gamma_{0,2}}}{\gamma_{0,1} \gamma_{0,2}\left(\left(s / g_{i}(s)\right)^{\gamma_{0,1}}-\left(s / g_{i}(s)\right)^{\gamma_{0,2}}\right)}
$$

for $s>K_{i} / L_{i}$ (see [34] and [24]). It is easily seen that the right-hand side of the expression in (3.64) is strictly positive, so that the candidate boundary $g_{i}(s)$ is a strictly increasing function on $\left(K_{i} / L_{i}, \infty\right)$. We also note that the functions in (3.63) do not depend on the boundary $g_{1-i}(s)$ directly, since the Markov chain $\Theta$ cannot leave the state $i$ when $\lambda_{i}=0$, for any $i=0,1$ fixed.

Substituting the expression $V_{i}(x, s)=L_{i} s-K_{i}$ into the equation of $(2.13)$ for $V_{1-i}(x, s)$, we obtain that its general solution has the form of (3.5), for all $g_{1-i}(s)<x<g_{i}(s)$, where $D_{1-i, k}(s)$ are some arbitrary functions, and $\beta_{1-i, 2}<0<1<\beta_{1-i, 1}$ are explicitly given by (3.6) above. Then, by applying the conditions of (2.14)-(2.15) to the function in (3.5), we obtain the equalities of (3.9)-(3.10), for $s>K_{1-i} / L_{1-i}$. In this case, solving the system of equations in (3.9)-(3.10), we obtain that the candidate value function $V_{1-i}\left(x, s ; g_{1-i}(s)\right)$ admits the representation of $(3.15)$, for $g_{1-i}(s)<x \leq g_{i}(s)$, with $D_{1-i, k}\left(s ; g_{1-i}(s)\right), k=1$, 2 , given by (3.16), where we set $L_{1-i}^{\prime}=L_{1-i}-\lambda_{1-i} L_{i} /\left(r+\lambda_{1-i}\right)$ and $K_{1-i}^{\prime}=K_{1-i}-\lambda_{1-i} K_{i} /\left(r+\lambda_{1-i}\right)$. We now recall that the general solution of the equation in $(2.13)$ for $V_{1-i}(x, s)$ has the form:

$$
V_{1-i}(x, s)=C_{1-i, 1}(s) x^{\beta_{1-i, 1}}+C_{1-i, 2}(s) x^{\beta_{1-i, 2}}+V_{i}\left(x, s ; g_{i}(s)\right)
$$


for $g_{i}(s)<x<s$, where $V_{i}\left(x, s ; g_{i}(s)\right)$ is given by (3.62) with (3.63). Moreover, using the fact that the function $V_{1-i}(x, s)$ in $(3.65)$ should be twice continuously differentiable at $g_{i}(s)$, for any $i=0,1$ fixed, and taking into account the conditions of (3.59)-(3.60), we get the equalities:

$$
\begin{aligned}
& C_{1-i, 1}(s) g_{i}^{\beta_{1-i, 1}}(s)+C_{1-i, 2}(s) g_{i}^{\beta_{1-i, 2}}(s)+L_{i} s-K_{i}=V_{1-i}\left(g_{i}(s), s ; g_{1-i}(s)\right) \\
& C_{1-i, 1}(s) \beta_{1-i, 1} g_{i}^{\beta_{1-i, 1}}(s)+C_{1-i, 2}(s) \beta_{1-i, 2} g_{i}^{\beta_{1-i, 2}}(s)=g_{i}(s) \partial_{x} V_{1-i}\left(g_{i}(s), s ; g_{1-i}(s)\right) \\
& C_{1-i, 1}(s) \beta_{1-i, 1}\left(\beta_{1-i, 1}-1\right) g_{i}^{\beta_{1-i, 1}}(s)+C_{1-i, 2}(s) \beta_{1-i, 2}\left(\beta_{1-i, 2}-1\right) g_{i}^{\beta_{1-i, 2}}(s) \\
& =g_{i}^{2}(s) \partial_{x x} V_{1-i}\left(g_{i}(s), s ; g_{1-i}(s)\right)
\end{aligned}
$$

for all $s>K_{1-i} / L_{1-i}$, where $V_{1-i}\left(x, s ; g_{1-i}(s)\right)$ is given by (3.15) with (3.16). Thus, solving the system of equations in (3.66)-(3.67), we obtain that the candidate value function admits the representation:

$$
V_{1-i}\left(x, s ; g_{i}(s), g_{1-i}(s)\right)=\sum_{k=1}^{2} C_{1-i, k}\left(s ; g_{i}(s), g_{1-i}(s)\right) x^{\beta_{1-i, k}}+V_{i}\left(x, s ; g_{i}(s)\right)
$$

for $g_{i}(s)<x \leq s$, with

$$
\begin{aligned}
& C_{1-i, k}\left(s ; g_{i}(s), g_{1-i}(s)\right) \\
& =\frac{\beta_{1-i, 3-k}\left(V_{1-i}\left(g_{i}(s), s ; g_{1-i}(s)\right)-L_{i} s+K_{i}\right)-g_{i}(s) \partial_{x} V_{1-i}\left(g_{i}(s), s ; g_{1-i}(s)\right)}{\left(\beta_{1-i, 3-k}-\beta_{1-i, k}\right) g_{i}^{\beta_{1-i, k}}(s)}
\end{aligned}
$$

for $k=1,2$. Then, by applying the condition of (2.16) to the function in (3.65), we obtain the equalities:

$$
C_{1-i, 1}^{\prime}(s) s^{\beta_{1-i, 1}}+C_{1-i, 2}^{\prime}(s) s^{\beta_{1-i, 2}}+\left(\partial_{s}+g_{i}^{\prime}(s) \partial_{g_{i}} V_{i}\right)\left(s, s ; g_{i}(s)\right)=0
$$

for $s>K_{1-i} / L_{1-i}$. Hence, by applying the condition of (3.71) to the functions in (3.70), we conclude that the candidate boundary satisfies the ordinary differential equation:

$$
\begin{aligned}
& g_{1-i}^{\prime}(s)=-\left(\left(\partial_{s}+g_{i}^{\prime}(s) \partial_{g_{i}}\right) V_{i}\left(s, s ; g_{i}(s)\right)\right. \\
& \left.+\left(\partial_{s}+g_{i}^{\prime}(s) \partial_{g_{i}}\right) C_{1-i, 1}\left(s ; g_{i}(s), g_{1-i}(s)\right) s^{\beta_{1-i, 1}}+\left(\partial_{s}+g_{i}^{\prime}(s) \partial_{g_{i}}\right) C_{1-i, 2}\left(s ; g_{i}(s), g_{1-i}(s)\right) s^{\beta_{1-i, 2}}\right) / \\
& \left(\partial_{g_{1-i}} C_{1-i, 1}\left(s ; g_{i}(s), g_{1-i}(s)\right) s^{\beta_{1-i, 1}}+\partial_{g_{1-i}} C_{1-i, 2}\left(s ; g_{i}(s), g_{1-i}(s)\right) s^{\beta_{1-i, 2}}\right)
\end{aligned}
$$

for $s>K_{1-i} / L_{1-i}$. It is seen that the system of equations in (3.64) and (3.72) is degenerated in the sense that the candidate boundary $g_{i}(s)$ solves the single first-order nonlinear ordinary differential equation in (3.64), while the boundary $g_{1-i}(s)$ can be either determined from the first-order nonlinear ordinary differential equation in (3.72) or expressed in terms of $g_{i}(s)$, by means of the arithmetic power equation in (3.68), for any $i=0,1$ fixed.

In order to characterise the asymptotic behaviour of the candidate boundaries $g_{j}(s), j=$ 0,1 , we observe that the expressions in (3.15)-(3.16) and (3.69)-(3.70) imply the representations of (3.23) and

$$
V_{i}\left(s, s ; g_{i}(s)\right) / s=\widetilde{C}_{i, 1}\left(s ; h_{i}(s)\right) h_{i}^{-\gamma_{0,1}}(s)+\widetilde{C}_{i, 2}\left(s ; h_{i}(s)\right) h_{i}^{-\gamma_{0,2}}(s)
$$


as well as

$$
\begin{aligned}
& V_{1-i}\left(s, s ; g_{i}(s), g_{1-i}(s)\right) / s \\
& =\sum_{k=1}^{2}\left(\widetilde{C}_{1-i, k}\left(s ; h_{i}(s), h_{1-i}(s)\right) h_{1-i}^{-\beta_{1-i, k}}(s)+\widetilde{C}_{i, k}\left(s ; h_{i}(s)\right) h_{1-i}^{-\gamma_{0} k}(s)\right)
\end{aligned}
$$

with some appropriately chosen $\widetilde{C}_{i, k}\left(s ; h_{i}(s)\right)$ and $\widetilde{C}_{1-i, k}\left(s ; h_{i}(s), h_{1-i}(s)\right)$, for $i=0,1$ and $k=1,2$, which are of constant signs as $s \rightarrow \infty$, where we recall that $h_{j}(s)=g_{j}(s) / s$, for all $s>K_{j} / L_{j}$ and every $j=0,1$. Then, following the arguments from the end of part (i) of Subsection 3.1, we may therefore conclude that the candidate boundary $g_{1-i}(s)$ should satisfy the conditions of (3.25) as well as the properties:

$$
g_{1-i}\left(\left(K_{1-i} / L_{1-i}\right)+\right)=0 \quad \text { and } \quad g_{1-i}(s) \sim A_{1-i}\left(L_{1-i} s-K_{1-i}\right)^{1 / \beta_{1-i, 1}} \text { as } \quad s \downarrow K_{1-i} / L_{1-i}
$$

with some constant $A_{1-i}>0$ which is also specified by means of the condition of (3.25) above. It also follows from the arguments of the end of part (i) of Subsection 3.1 above that there exist maximal solutions $g_{j}^{*}(s), j=0,1$, of the first-order nonlinear ordinary differential equations in (3.64) and (3.72) such that the inequalities $0<g_{j}^{*}(s) \leq a_{j}^{*} s$ hold, for $j=0,1$.

(ii) In order to find the candidate value function $V_{1-i}(x, s)$ in the region $K_{i} / L_{i}<x \leq s \leq$ $K_{1-i} / L_{1-i}$, we recall that the general solution of (2.13) is given by (3.5), for all $0<x<g_{i}(s)$ and any $i=0,1$ fixed. In this case, following the arguments of part (ii) of Subsection 3.1 above and solving the equation in (3.68) with $C_{1-i, k}\left(s ; g_{i}(s), g_{1-i}(s)\right), k=1,2$, from (3.70) and $V_{i}\left(x, s ; g_{i}(s)\right)$ from (3.62) with (3.63), we obtain that the candidate solution $V_{1-i}\left(x, s ; g_{i}(s)\right)$ takes the form of $(3.27)$ with $D_{1-i, 1}\left(s ; g_{i}(s)\right)$ given by:

$$
\left.D_{1-i, 1}\left(s ; g_{i}(s)\right)=r\left(L_{i} s-K_{i}\right) /\left(\left(r+\lambda_{1-i}\right)\left(1-\beta_{1-i, 1}\left(\beta_{1-i, 1}-1\right)\right)\right) g_{i}^{\beta_{1-i, 1}}(s)\right)
$$

for $0<x \leq g_{i}(s), i=0,1$. We also note that, by virtue of the arguments similar to the ones from the end of part (i) above, the candidate boundary $g_{i}(s)$ should satisfy the properties:

$$
g_{i}\left(\left(K_{i} / L_{i}\right)+\right)=0 \quad \text { and } \quad g_{i}(s) \sim A_{i}\left(L_{i} s-K_{i}\right)^{1 / \gamma_{0,1}} \quad \text { as } \quad s \downarrow K_{i} / L_{i}
$$

with some constant $A_{i}>0$ which is specified by means of the condition of (3.25) above.

(iii) By means of the same arguments as in part (iii) of Subsection 3.2, it is shown that the candidate value functions $V_{j}\left(x, s ; K_{i} / L_{i}\right), j=0,1$, take the form of (3.57), for all $0<x \leq s \leq$ $K_{i} / L_{i}$ and any $i=0,1$ fixed, where the functions $V_{j}\left(K_{i} / L_{i}, K_{i} / L_{i}\right)=V_{j}\left(K_{i} / L_{i}+, K_{i} / L_{i}+\right)$, $j=0,1$, are determined in parts (i)-(ii) above.

\section{Main results and proofs}

In this section, based on the facts proved above, we formulate and prove the main result of the paper. We recall here that $a_{i}^{*}$ is defined by $a_{i}^{*}=b_{i}^{*}(0,0)$ with $b_{i}^{*} \equiv b_{i}^{*}\left(K_{0}, K_{1}\right)$ determined from the expressions in (5.15) with (5.16), or (5.19) and (5.22), or (5.25) and (5.28), for every $i=0,1$ (see Theorem 5.1 below). 
Theorem 4.1 Let the process $(X, S)$ be given by (1.3)-(1.4) and (2.2) with $\sigma>0, \mu<r$, and $r>0$, and $\Theta$ be a two-state continuous-time Markov chain with the state space $\{0,1\}$ and transition intensities $\lambda_{i}>0, i=0,1$. Assume that $K_{i} / L_{i} \leq K_{1-i} / L_{1-i}$ holds, for any $i=0,1$ fixed. Then, the value function of the optimal stopping problem in (2.1) admits the representations:

$$
V_{i}^{*}(x, s)= \begin{cases}V_{i}\left(x, s ; g_{i}^{*}(s), g_{1-i}^{*}(s)\right), & \text { if } g_{i}^{*}(s)<x \leq s, \quad s>K_{1-i} / L_{1-i} \\ V_{i}\left(x, s ; g_{i}^{*}(s)\right), & \text { if } g_{i}^{*}(s)<x \leq s, \quad K_{i} / L_{i}<s \leq K_{1-i} / L_{1-i} \\ L_{i} s-K_{i}, & \text { if } 0<x \leq g_{i}^{*}(s), \quad s>K_{i} / L_{i} \\ V_{i}\left(x ; K_{i} / L_{i}\right), & \text { if } 0<x \leq s \leq K_{i} / L_{i}\end{cases}
$$

and

$$
V_{1-i}^{*}(x, s)= \begin{cases}V_{1-i}\left(x, s ; g_{i}^{*}(s), g_{1-i}^{*}(s)\right), & \text { if } g_{i}^{*}(s)<x \leq s, \quad s>K_{1-i} / L_{1-i} \\ V_{1-i}\left(x, s ; g_{1-i}^{*}(s)\right), & \text { if } g_{1-i}^{*}(s)<x \leq g_{i}^{*}(s), \quad s>K_{1-i} / L_{1-i} \\ L_{1-i} s-K_{1-i}, & \text { if } 0<x \leq g_{1-i}^{*}(s), \quad s>K_{1-i} / L_{1-i} \\ V_{1-i}\left(x, s ; g_{i}^{*}(s)\right), & \text { if } K_{i} / L_{i}<x \leq s \leq K_{1-i} / L_{1-i} \\ V_{1-i}\left(x ; K_{i} / L_{i}\right), & \text { if } 0<x \leq s \leq K_{i} / L_{i}\end{cases}
$$

and the optimal stopping time has the form:

$$
\tau^{*}=\inf \left\{t \geq 0 \mid X_{t} \leq g_{\Theta_{t}}^{*}\left(S_{t}\right)\right\}
$$

where the candidate value functions and (non-decreasing) boundaries are specified as follows:

(i): if $\lambda_{i}>0$, for every $i=0,1$, and $s>K_{1-i} / L_{1-i}$, then $V_{j}\left(x, s ; g_{i}(s), g_{1-i}(s)\right), j=$ 0,1 , are given by (3.17), for $g_{i}(s)<x \leq s$, with $C_{j, k}\left(s ; g_{i}(s), g_{1-i}(s)\right), j=0,1, k=1,2$, from (3.18)-(3.19), and $V_{1-i}\left(x, s ; g_{1-i}(s)\right)$ is given by (3.15), for $g_{1-i}(s)<x \leq g_{i}(s)$, with $D_{1-i, k}\left(s ; g_{1-i}(s)\right), k=1,2$, from (3.16), while $g_{j}^{*}(s), j=0,1$, are the maximal solutions of the (first-order nonlinear) ordinary differential equations in (3.14) with (3.20)-(3.21) and (3.22) such that $g_{j}^{*}(s) \leq a_{j}^{*} s, j=0,1$, for $s>K_{1-i} / L_{1-i}$, satisfying the conditions of (3.25) and the properties in (3.26);

(ii): if $\lambda_{i}>0$, for every $i=0,1$, and $0<s \leq K_{1-i} / L_{1-i}$, then $V_{1-i}\left(x, s ; g_{i}(s)\right)$ is given by (3.27), for $0<x \leq g_{i}(s)$, with $D_{1-i, 1}\left(s ; g_{i}(s)\right)$ from (3.28), and $V_{j}\left(x, s ; g_{i}(s)\right), j=0,1$, are given by (3.17), for $g_{i}(s)<x \leq s$, with $C_{j, k}\left(s ; g_{i}(s)\right), j=0,1, k=1,2$, determined as in (3.18)-(3.19) with $D_{1-i, 1}\left(s ; g_{i}(s)\right)$ from (3.28) and $D_{1-i, 2}\left(s ; g_{i}(s)\right)=0$, while $g_{i}^{*}(s)$ is the maximal solution of the ordinary differential equation in (3.14) such that $g_{i}^{*}(s) \leq a_{i}^{*} s$ under $j=i$ with $C_{j, k}^{\prime}\left(s ; g_{i}(s)\right), k=1,2$, determined as in (3.20)-(3.21) with $D_{1-i, 1}^{\prime}\left(s ; g_{i}(s)\right)$ from (3.29) and $D_{1-i, 2}^{\prime}\left(s ; g_{i}(s)\right)=0$, for $K_{i} / L_{i}<s<K_{1-i} / L_{1-i}$ such that $g_{i}^{*}\left(\left(K_{1-i} / L_{1-i}\right)-\right)=$ $g_{i}^{*}\left(\left(K_{1-i} / L_{1-i}\right)+\right)$, as well as $V_{j}\left(x ; K_{i} / L_{i}\right), j=0,1$, are given by (3.33), for $0<x \leq s \leq$ $K_{i} / L_{i}$, with $C_{j, 1}\left(K_{i} / L_{i}\right), j=0,1$, from (3.34);

(iii): if $\lambda_{i}>\lambda_{1-i}=0$, for any $i=0,1$ fixed, and $s>K_{1-i} / L_{1-i}$, then $V_{1-i}\left(x, s ; g_{1-i}(s)\right)$ is given by (3.39), for $g_{1-i}(s)<x \leq s$, with $C_{1-i, k}\left(s ; g_{1-i}(s)\right), k=1,2$, from (3.40), and $V_{i}\left(x, s ; g_{i}(s), g_{1-i}(s)\right)$ is given by (3.46), for $g_{i}(s)<x \leq s$, with $C_{i, k}\left(s ; g_{i}(s), g_{1-i}(s)\right), k=$ 1,2 , from (3.47), while $g_{j}^{*}(s), j=0,1$, are the maximal solutions of the ordinary differential equations in (3.41) and (3.48) such that $g_{1-i}^{*}(s) \leq a_{1-i}^{*} s$, for $s>K_{1-i} / L_{1-i}$, satisfying the conditions of (3.25) and the properties in (3.51); 
(iv): if $\lambda_{i}>\lambda_{1-i}=0$, for any $i=0,1$ fixed, and $0<s \leq K_{1-i} / L_{1-i}$, then $V_{1-i}\left(x ; K_{1-i} / L_{1-i}\right)$ is given by (3.52), for $0<x \leq s \leq K_{1-i} / L_{1-i}$, and $V_{i}\left(x, s ; g_{i}(s)\right)$ is given by (3.53), for $g_{i}(s)<x \leq s$, with $C_{i, k}\left(s ; g_{i}(s)\right), k=1,2$, from (3.54), while $g_{i}^{*}(s)$ is the maximal solution of the ordinary differential equation in (3.55) such that $g_{i}^{*}(s) \leq a_{i}^{*} s$, for $K_{i} / L_{i}<s<K_{1-i} / L_{1-i}$, satisfying the conditions of (3.25) and the properties in (3.56), as well as $V_{j}\left(x ; K_{i} / L_{i}\right), j=0,1$, are given by (3.57), for $0<x \leq s \leq K_{i} / L_{i}$;

(v): if $\lambda_{1-i}>\lambda_{i}=0$, for any $i=0,1$ fixed, then $V_{i}\left(x, s ; g_{i}(s)\right)$ is given by (3.62), for $g_{i}(s)<x \leq s$, with $C_{i, k}\left(s ; g_{i}(s)\right), k=1,2$, from (3.63), while $g_{i}^{*}(s)$ is the maximal solution of the ordinary differential equation in (3.64) such that $g_{i}^{*}(s) \leq a_{i}^{*} s$, for $s>K_{i} / L_{i}$, satisfying the conditions of (3.25) and the properties in (3.77), $V_{1-i}\left(x, s ; g_{1-i}(s)\right)$ is given by (3.15), for $g_{1-i}(s)<x \leq g_{i}(s)$, with $D_{1-i, k}\left(s ; g_{1-i}(s)\right), k=1,2$, from (3.16), $V_{1-i}\left(x, s ; g_{i}(s), g_{1-i}(s)\right)$ is given by (3.69), for $g_{i}(s)<x \leq s$, with $C_{1-i, k}\left(s ; g_{i}(s), g_{1-i}(s)\right), k=1,2$, from (3.70), while $g_{1-i}^{*}(s)$ is the maximal solution of the ordinary differential equation in (3.72) such that $g_{1-i}^{*}(s) \leq a_{1-i}^{*} s$, for $s>K_{1-i} / L_{1-i}$, satisfying the conditions of (3.25) and the properties in (3.75), and $V_{1-i}\left(x, s ; g_{i}(s)\right)$ is given by (3.27), for $0<x \leq g_{i}(s)$ and $K_{i} / L_{i}<x \leq s<$ $K_{1-i} / L_{1-i}$, with $D_{1-i, 1}\left(s ; g_{i}(s)\right)$ from (3.76), as well as $V_{j}\left(x ; K_{i} / L_{i}\right), j=0,1$, are given by (3.57), for $0<x \leq s \leq K_{i} / L_{i}$.

Since all the assertions formulated above are proved using similar arguments, we only give a proof for the general case $\lambda_{i}>0, i=0,1$, for the intensities of the continuous-time Markov chain $\Theta$ considered in parts (i) and (ii) of Theorem 4.1.

Proof In order to verify the assertions stated above, it remains for us to show that the functions defined in the right-hand sides of (4.1) and (4.2) coincide with the value functions in (2.1) and that the stopping time $\tau^{*}$ in (4.3) is optimal with the boundaries $g_{j}^{*}(s), j=0,1$, specified above. For this purpose, let the components of the couple $\left(g_{j}(s), g_{1-j}(s)\right)$ be any solution of the (system of) first-order nonlinear ordinary differential equations in (3.14) with (3.20)-(3.21) and (3.22) such that $0<g_{j}(s) \leq a_{j}^{*} s$ holds, for $s>K_{j} / L_{j}$, and satisfying the condition of (3.25), for any $j=0,1$ fixed. We thus denote by $V_{i}^{\left(g_{j}, g_{1-j}\right)}(x, s)$ the right-hand sides of the expressions in (4.1) and (4.2) associated with $\left(g_{j}(s), g_{1-j}(s)\right), j=0,1$, for any $i=0,1$ fixed. Then, it is shown by means of straightforward calculations from the previous section that the functions $V_{i}^{\left(g_{j}, g_{1-j}\right)}(x, s), i, j=0,1$, solve the system of (2.13) with (2.17)-(2.19) and satisfy the conditions of (2.14)-(2.16). We also recall the fact that the functions $V_{i}^{\left(g_{j}, g_{1-j}\right)}(x, s)$ are $C^{2,1}$ on the closures $\bar{C}_{i}$ of $C_{i}$ and are equal to $L_{i} s-K_{i}$ on $D_{i}$, which are defined as $\bar{C}_{i}^{*}, C_{i}^{*}$ and $D_{i}^{*}$ in (2.3) with (2.7) and (2.8) with $g_{i}(s)$ instead of $g_{i}^{*}(s)$, for any $i=0,1$, respectively. Hence, using the assumption that the boundaries $g_{i}(s), i=0,1$, are continuously differentiable as well as taking into account the fact that the processes $(X, S)$ and $\Theta$ are independent, by applying the change-of-variable formula from [36; Theorem 3.1] to the process $e^{-r t} V_{\Theta_{t}}^{\left(g_{j}, g_{1-j}\right)}\left(X_{t}, S_{t}\right)$ (see also [39; Chapter II, Section 3.5] for a summary of the related results and further references), 
we obtain the expression:

$$
\begin{gathered}
e^{-r t} V_{\Theta_{t}}^{\left(g_{j}, g_{1-j}\right)}\left(X_{t}, S_{t}\right)=V_{i}^{\left(g_{j}, g_{1-j}\right)}(x, s)+\int_{0}^{t} e^{-r u} \partial_{s} V_{\Theta_{u}}^{\left(g_{j}, g_{1-j}\right)}\left(X_{u}, S_{u}\right) I\left(X_{u}=S_{u}\right) d S_{u} \\
+\int_{0}^{t} e^{-r u}\left(\mathbb{L} V_{\Theta_{u}}^{\left(g_{j}, g_{1-j}\right)}-\left(r+\lambda_{\Theta_{u}}\right) V_{\Theta_{u}}^{\left(g_{j}, g_{1-j}\right)}+\lambda_{\Theta_{u}} V_{1-\Theta_{u}}^{\left(g_{j}, g_{1-j}\right)}\right)\left(X_{u}, S_{u}\right) \\
\times I\left(X_{u} \neq g_{j}\left(S_{u}\right), j=0,1, X_{u} \neq S_{u}\right) d u+M_{t}
\end{gathered}
$$

for all $t \geq 0$ and every $j=0,1$. Here, the process $M=\left(M_{t}\right)_{t \geq 0}$ defined by:

$$
\begin{aligned}
M_{t}= & \int_{0}^{t} e^{-r u} \partial_{x} V_{\Theta_{u}}^{\left(g_{j}, g_{1-j}\right)}\left(X_{u}, S_{u}\right) I\left(X_{u} \neq S_{u}\right) \sigma X_{u} d B_{u} \\
& +\int_{0}^{t} e^{-r u}\left(V_{1}^{\left(g_{j}, g_{1-j}\right)}\left(X_{u}, S_{u}\right)-V_{0}^{\left(g_{j}, g_{1-j}\right)}\left(X_{u}, S_{u}\right)\right) I\left(X_{u} \neq S_{u}\right) d N_{u}
\end{aligned}
$$

with $N=\left(N_{t}\right)_{t \geq 0}$ given by:

$$
N_{t}=\Theta_{t}-\int_{0}^{t}\left(\lambda_{0}\left(1-\Theta_{u-}\right)+\lambda_{1} \Theta_{u-}\right) d u
$$

is a local martingale with respect to the probability measure $P_{x, s, i}$, and $I(\cdot)$ denotes the indicator function. Note that, since the time spent by the process $(X, S)$ at the boundary surfaces $\left\{(x, s) \in E \mid x=g_{i}(s)\right\}, i=0,1$, as well as at the diagonal $\left\{(x, s) \in \mathbb{R}^{2} \mid 0<x=s\right\}$ is of Lebesgue measure zero, the indicators in the third line of the formula in (4.4) as well as in the expression of (4.5) can be ignored. Moreover, since the component $S$ increases only when the process $(X, S)$ is located on the diagonal $\left\{(x, s) \in \mathbb{R}^{2} \mid 0<x=s\right\}$, the indicator in the first line of (4.4) can be set equal to one.

By using straightforward calculations and the arguments from the previous section, it is verified that $\left(\mathbb{L} V_{i}^{\left(g_{j}, g_{1-j}\right)}-\left(r+\lambda_{i}\right) V_{i}^{\left(g_{j}, g_{1-j}\right)}+\lambda_{i} V_{1-i}^{\left(g_{j}, g_{1-j}\right)}\right)(x, s) \leq 0$ holds, for all $(x, s, i) \in$ $E \times\{0,1\}$ such that $x \neq g_{i}(s), i=0,1$, and $x \neq s$. Furthermore, it is shown by means of standard arguments that the properties in (2.18)-(2.19) also hold, which together with the conditions of (2.14)-(2.17) imply that the inequalities $V_{i}^{\left(g_{j}, g_{1-j}\right)}(x, s) \geq L_{i} s-K_{i}$ are satisfied, for all $(x, s, i) \in E \times\{0,1\}$ and every $j=0,1$. Let $\left(\varkappa_{n}\right)_{n \in \mathbb{N}}$ be the localising sequence of stopping times for the process $M$ from (4.5) such that $\varkappa_{n}=\inf \left\{t \geq 0|| M_{t} \mid \geq n\right\}$, for each $n \in \mathbb{N}$. It therefore follows from the expression in (4.4) that the inequalities:

$$
\begin{gathered}
e^{-r\left(\tau \wedge \varkappa_{n}\right)}\left(\left(1-\Theta_{\tau \wedge \varkappa_{n}}\right)\left(L_{0} S_{\tau \wedge \varkappa_{n}}-K_{0}\right)+\Theta_{\tau \wedge \varkappa_{n}}\left(L_{1} S_{\tau \wedge \varkappa_{n}}-K_{1}\right)\right) \\
\leq e^{-r\left(\tau \wedge \varkappa_{n}\right)} V_{\Theta_{\tau \wedge \varkappa_{n}}^{\left(g_{j}, g_{1-j}\right)}}\left(X_{\tau \wedge \varkappa_{n}}, S_{\tau \wedge \varkappa_{n}}\right) \leq V_{i}^{\left(g_{j}, g_{1-j}\right)}(x, s)+M_{\tau \wedge \varkappa_{n}}
\end{gathered}
$$

hold, for any finite stopping time $\tau$ with respect to the natural filtration of $(X, \Theta)$ and each $n \in \mathbb{N}$, and any $j=0,1$ fixed. Then, taking the expectation with respect to $P_{x, s, i}$ in (4.7), by means of Doob's optional sampling theorem (see, e.g. [31; Chapter III, Theorem 3.6] or [41; Chapter II, Theorem 3.2]), we get:

$$
\begin{aligned}
& E_{x, s, i}\left[e^{-r\left(\tau \wedge \varkappa_{n}\right)}\left(\left(1-\Theta_{\tau \wedge \varkappa_{n}}\right)\left(L_{0} S_{\tau \wedge \varkappa_{n}}-K_{0}\right)+\Theta_{\tau \wedge \varkappa_{n}}\left(L_{1} S_{\tau \wedge \varkappa_{n}}-K_{1}\right)\right)\right] \\
& \leq E_{x, s, i}\left[e^{-r\left(\tau \wedge \varkappa_{n}\right)} V_{\Theta_{\tau \wedge \varkappa_{n}}^{\left(g_{j}, g_{1-j}\right)}}\left(X_{\tau \wedge \varkappa_{n}}, S_{\tau \wedge \varkappa_{n}}\right)\right] \leq V_{i}^{\left(g_{j}, g_{1-j}\right)}(x, s)+E_{x, s, i}\left[M_{\tau \wedge \varkappa_{n}}\right]=V_{i}^{\left(g_{j}, g_{1-j}\right)}(x, s)
\end{aligned}
$$


for all $(x, s, i) \in E \times\{0,1\}$ and each $n \in \mathbb{N}$, and any $j=0,1$ fixed. Hence, letting $n$ go to infinity and using Fatou's lemma, we obtain from the expressions in (4.8) that the inequalities:

$$
\begin{aligned}
& E_{x, s, i}\left[e^{-r \tau}\left(\left(1-\Theta_{\tau}\right)\left(L_{0} S_{\tau}-K_{0}\right)+\Theta_{\tau}\left(L_{1} S_{\tau}-K_{1}\right)\right)\right] \\
& \leq E_{x, s, i}\left[e^{-r \tau} V_{\Theta_{\tau}}^{\left(g_{j}, g_{1-j}\right)}\left(X_{\tau}, S_{\tau}\right)\right] \leq V_{i}^{\left(g_{j}, g_{1-j}\right)}(x, s)
\end{aligned}
$$

hold, for any finite stopping time $\tau$, and all $(x, s, i) \in E \times\{0,1\}$, and any $j=0,1$ fixed. Thus, taking first the supremum over all stopping times $\tau$ and then the infimum over all couples of boundaries $\left(g_{j}, g_{1-j}\right)$ in the expressions of $(4.9)$, we conclude that the inequalities:

$$
\begin{aligned}
& E_{x, s, i}\left[e^{-r \tau^{*}}\left(\left(1-\Theta_{\tau^{*}}\right)\left(L_{0} S_{\tau^{*}}-K_{0}\right)+\Theta_{\tau^{*}}\left(L_{1} S_{\tau^{*}}-K_{1}\right)\right)\right] \\
& \leq \inf _{\left(g_{j}, g_{1-j}\right)} V_{i}^{\left(g_{j}, g_{1-j}\right)}(x, s)=V_{i}^{\left(g_{j}^{*}, g_{1-j}^{*}\right)}(x, s)
\end{aligned}
$$

are satisfied, for $(x, s, i) \in E \times\{0,1\}$, where the components of the couple $\left(g_{j}^{*}(s), g_{1-j}^{*}(s)\right)$, $j=0,1$, are the largest possible solutions of the (system of) first-order nonlinear ordinary differential equations in (3.14) with (3.20)-(3.21) and (3.22) as well as satisfying the condition of (3.25) and such that $0<g_{j}^{*}(s) \leq a_{j}^{*} s$ holds, for all $s>K_{j} / L_{j}$, and any $j=0,1$ fixed. Taking into account the fact that the function $V_{i}^{\left(g_{j}, g_{1-j}\right)}(x, s)$ is (strictly) decreasing in the couple of functions $\left(g_{j}(s), g_{1-j}(s)\right)$, we see that the infimum in (4.10) is attained over any sequence of solutions $\left(g_{j, m}(s), g_{1-j, m}(s)\right)_{m \in \mathbb{N}}$ to the system in (3.14) with (3.20)-(3.21) and (3.22) as well as satisfying the condition of (3.25) and such that $g_{j, m}(s) \uparrow g_{j}^{*}(s)$ as $m \rightarrow \infty$, for each $s>K_{j} / L_{j}$ fixed, and every $j=0,1$. It follows from the classical results on the (local) uniqueness of solutions to the (sysemts of) first-order nonlinear ordinary differential equations in (3.14) with (3.20)-(3.21) and (3.22) that no distinct solutions intersect, so that the sequence $\left(g_{j, m}(s)\right)_{m \in \mathbb{N}}$ is increasing and the limit $g_{j}^{*}(s)=\lim _{m \rightarrow \infty} g_{j, m}(s)$ exists, for each $s>K_{j} / L_{j}$ fixed, and every $j=0,1$. Since the inequalities in (4.9) hold for $\left(g_{j}^{*}(s), g_{1-j}^{*}(s)\right)$ too, we see that the expression in (4.10) holds, for $\left(g_{j}^{*}(s), g_{1-j}^{*}(s)\right)$ and $(x, s, i) \in E \times\{0,1\}$, as well. We also note from the inequality in (4.8) that the function $V_{i}^{\left(g_{j}, g_{1-j}\right)}(x, s)$ is superharmonic for the Markov process $(X, S, \Theta)$ on $E \times\{0,1\}$. Hence, by using the facts that $V_{i}^{\left(g_{j}, g_{1-j}\right)}(x, s)$ is decreasing in $\left(g_{j}(s), g_{1-j}(s)\right)$ such that $0<g_{j}(s) \leq a_{j}^{*} s$, and the inequality $V_{i}^{\left(g_{j}, g_{1-j}\right)}(x, s) \geq L_{i} s-K_{i}$ holds, for all $(x, s, i) \in E \times\{0,1\}$, we observe that the selection of the maximal admissible solutions $g_{j}^{*}(s), j=0,1$, as the largest possible solutions of the equations in (3.14) which stay strictly below the lines $x=a_{j}^{*} s$, for all $s>K_{j} / L_{j}$, and any $j=0,1$ fixed, is equivalent to the implementation of the superharmonic characterisation of the payoff function (see [35] and [39; Chapter I and Chapter V, Section 17] for the associated maximality principle).

In order to prove the fact that the components of the couple $\left(g_{j}^{*}(s), g_{1-j}^{*}(s)\right)$ provide the optimal boundaries, we consider a sequence of stopping times $\tau_{m}, m \in \mathbb{N}$, which are defined as in (4.3) with $\left(g_{j, m}(s), g_{1-j, m}(s)\right)$ instead of $\left(g_{j}^{*}(s), g_{1-j}^{*}(s)\right)$, where the components of the couple $\left(g_{j, m}(s), g_{1-j, m}(s)\right)$ are solutions to the (system of) first-order ordinary differential equations in (3.14) with (3.20)-(3.21) and (3.22) as well as satisfying the condition of (3.25) and such that $g_{j, m}(s) \uparrow g_{j}^{*}(s)$ as $m \rightarrow \infty$, for each $s>K_{j} / L_{j}$ fixed, and every $j=0,1$. Then, by virtue of the fact that the functions $V_{i}^{\left(g_{j, m}, g_{1-j, m}\right)}(x, s)$ from the right-hand sides of the expressions in (4.1)-(4.2) associated with the boundaries $g_{j, m}(s), j=0,1, m \in \mathbb{N}$, satisfy the conditions of 
(2.13) and (2.14), for any $j=0,1$ fixed, and taking into account the structure of $\tau^{*}$ in (4.3), it follows from the expression which is equivalent to the one in (4.4) that the equalities:

$$
\begin{aligned}
& e^{-r\left(\tau_{m} \wedge \varkappa_{n}\right)}\left(\left(1-\Theta_{\tau_{m} \wedge \varkappa_{n}}\right)\left(L_{0} S_{\tau_{m} \wedge \varkappa_{n}}-K_{0}\right)+\Theta_{\tau_{m} \wedge \varkappa_{n}}\left(L_{1} S_{\tau_{m} \wedge \varkappa_{n}}-K_{1}\right)\right) \\
& =e^{-r\left(\tau_{m} \wedge \varkappa_{n}\right)} V_{\Theta_{\tau_{m} \wedge \varkappa_{n}}^{\left(g_{j, m}, g_{1-j, m}\right)}}\left(X_{\tau_{m} \wedge \varkappa_{n}}, S_{\tau_{m} \wedge \varkappa_{n}}\right)=V_{i}^{\left(g_{j, m}, g_{1-j, m}\right)}(x, s)+M_{\tau_{m} \wedge \varkappa_{n}}
\end{aligned}
$$

hold, for all $(x, s, i) \in E \times\{0,1\}$ and each $n, m \in \mathbb{N}$, and any $j=0,1$ fixed. Observe that, by virtue of the arguments from [43; pages 635-636], the property:

$$
E_{x, s, i}\left[\sup _{t \geq 0} e^{-r\left(\tau^{*} \wedge t\right)} S_{\tau^{*} \wedge t}\right]=E_{x, s, i}\left[\sup _{t \geq 0} e^{-r\left(\tau^{*} \wedge t\right)} X_{\tau^{*} \wedge t}\right]<\infty
$$

holds, for all $(x, s, i) \in E \times\{0,1\}$, as well as the variable $e^{-r \tau^{*}} S_{\tau^{*}}$ is bounded on the event $\left\{\tau^{*}=\infty\right\}$. We also note that, by analysing the asymptotic behavior of $g_{j}^{*}(s), j=0,1$, at infinity, it is verified that $P_{x, s, i}\left(\tau^{*}<\infty\right)=1$, for all $(x, s, i) \in E \times\{0,1\}$. Hence, letting $m$ and $n$ go to infinity and using the condition of (2.14) as well as the property $\tau_{m} \downarrow \tau^{*}\left(P_{x, s, i}\right.$-a.s. $)$ as $m \rightarrow \infty$, we can apply the Lebesgue dominated convergence theorem to the appropriate (diagonal) subsequence in the expression of (4.11) to obtain the equality:

$$
E_{x, s, i}\left[e^{-r \tau^{*}}\left(\left(1-\Theta_{\tau^{*}}\right)\left(L_{0} S_{\tau^{*}}-K_{0}\right)+\Theta_{\tau^{*}}\left(L_{1} S_{\tau^{*}}-K_{1}\right)\right)\right]=V_{i}^{\left(g_{j}^{*}, g_{1-j}^{*}\right)}(x, s)
$$

for all $(x, s, i) \in E \times\{0,1\}$ and any $j=0,1$ fixed, which together with the inequalities in (4.9) directly implies the desired assertion.

\section{Solutions to the problem with floating sunk costs}

In this section, we obtain an explicit solution of the optimal stopping problem of (1.2) which is related to the floating sunk costs real switching lookback option pricing problem. Although, by means of the change-of-measure arguments of [44], the problem of (1.2) can be reduced to an optimal stopping problem for a two-dimensional Markov process having the Markov chain $\Theta$ as one of the state-space components, we prefer to follow the arguments of [43] and solve it as a three-dimensional optimal stopping problem, by using the arguments of Sections 2-4 above applied to the optimal stopping problem of (2.1), in order to spare some space of the paper.

5.1 The optimal stopping and free-boundary problem. It is seen that the problem of (1.2) can be embedded to the optimal stopping problem for the (time-homogeneous strong) Markov process $(X, S, \Theta)=\left(X_{t}, S_{t}, \Theta_{t}\right)_{t \geq 0}$ with the value function:

$$
W_{i}^{*}(x, s)=\sup _{\zeta} E_{x, s, i}\left[e^{-r \zeta}\left(\left(1-\Theta_{\zeta}\right)\left(L_{0} S_{\zeta}-K_{0} X_{\zeta}\right)+\Theta_{\zeta}\left(L_{1} S_{\zeta}-K_{1} X_{\zeta}\right)\right)\right]
$$

for some $L_{i}>0$ and $K_{i} \geq 0, i=0,1$, fixed, where the supremum is taken over all stopping times $\zeta$ with respect to $\left(\mathcal{G}_{t}\right)_{t \geq 0}$. For simplicity of presentation, we further assume that $L_{i} \geq K_{i}$ holds, for every $i=0,1$. It can be shown by means of the same arguments as in Subsection 2.2 above that the optimal stopping time in the problem of (5.1) has the structure:

$$
\zeta^{*}=\inf \left\{t \geq 0 \mid X_{t} \leq b_{\Theta_{t}}^{*} S_{t}\right\}
$$


with some numbers $0<b_{i}^{*}<1, i=0,1$, to be determined.

In order to find analytic expressions for the unknown value functions $W_{i}^{*}(x, s)$ from $(5.1)$ and the unknown boundaries $b_{i}^{*} s, i=0,1$, from (5.2), we can formulate the following freeboundary problem:

$$
\begin{aligned}
& \left(\mathbb{L} W_{i}-\left(r+\lambda_{i}\right) W_{i}\right)(x, s)=-\lambda_{i} W_{1-i}(x, s) \quad \text { for } \quad b_{i} s<x<s \\
& \left.W_{i}(x, s)\right|_{x=\left(b_{i} s\right)+}=L_{i} s-K_{i} b_{i} s \quad \text { (instantaneous stopping) } \\
& \left.\partial_{x} W_{i}(x, s)\right|_{x=\left(b_{i} s\right)+}=-K_{i} \quad(\text { smooth fit) } \\
& \left.\partial_{s} W_{i}(x, s)\right|_{x=s-}=0 \quad(\text { normal reflection) } \\
& W_{i}(x, s)=L_{i} s-K_{i} x \quad \text { for } \quad 0<x<b_{i} s \\
& W_{i}(x, s)>L_{i} s-K_{i} x \quad \text { for } \quad b_{i} s<x \leq s \\
& \left(\mathbb{L} W_{i}-\left(r+\lambda_{i}\right) W_{i}\right)(x, s)<-\lambda_{i} W_{1-i}(x, s) \text { for } 0<x<b_{i} s
\end{aligned}
$$

where the conditions of (5.4)-(5.6) are satisfied, for all $s>0$ and any $i=0,1$ fixed. Note that by virtue of the structure of (5.1) and (5.2) it is easily seen that $0<b_{j}^{*} \leq L_{j} / K_{j}$, for every $j=0,1$.

5.2 Solution to the free-boundary problem. Let us now present the solution of the free-boundary problem in (5.3)-(5.9).

(i) Let us first assume that $\lambda_{i}>0, i=0,1$, holds. In this case, by using straightforward calculations from part (i) of Subsection 3.1, it can be shown that the solution of the system in (5.3)-(5.9) takes the form:

$$
W_{1-i}\left(x, s ; b_{1-i}^{*}\right)=\sum_{k=1}^{2} \widehat{D}_{1-i, k}\left(s ; b_{1-i}^{*}\right) x^{\beta_{1-i, k}}+\frac{\lambda_{1-i} L_{i} s}{r+\lambda_{1-i}}-\frac{\lambda_{1-i} K_{i} x}{r+\lambda_{1-i}-\mu}
$$

for $0<b_{1-i}^{*} s<x \leq b_{i}^{*} s$, with

$$
\widehat{D}_{1-i, k}\left(s ; b_{1-i}^{*}\right)=\frac{\beta_{1-i, 3-k} L_{1-i}^{\prime} s+\left(1-\beta_{1-i, 3-k}\right) K_{1-i}^{\prime \prime} b_{1-i}^{*} s}{\left(\beta_{1-i, 3-k}-\beta_{1-i, k}\right)\left(b_{1-i}^{*} s\right)^{\beta_{1-i, k}}}
$$

for $k=1,2$, where we recall that $L_{1-i}^{\prime}=L_{1-i}-\lambda_{1-i} L_{i} /\left(r+\lambda_{1-i}\right)$ and set $K_{1-i}^{\prime \prime}=K_{1-i}-$ $\lambda_{1-i} K_{i} /\left(r+\lambda_{1-i}-\mu\right)$, and

$$
W_{j}\left(x, s ; b_{i}^{*}, b_{1-i}^{*}\right)=\sum_{k=1}^{2}\left(\lambda_{j} \widehat{C}_{j, k}\left(s ; b_{i}^{*}, b_{1-i}^{*}\right) x^{\gamma_{j, k}}-(-1)^{j} \widehat{C}_{1-j, k}\left(s ; b_{i}^{*}, b_{1-i}^{*}\right) x^{\gamma_{1-j, k}}\right)
$$

for $0<b_{i}^{*} s<x<s, i=0,1$, where the functions $\widehat{C}_{j, k}\left(s ; b_{i}^{*}, b_{1-i}^{*}\right)$, for $j=0,1$ and $k=1,2$, admit the representations:

$$
\begin{aligned}
& \widehat{C}_{i, k}\left(s ; b_{i}^{*}, b_{1-i}^{*}\right)=(-1)^{i} /\left(\left(\lambda_{0} \lambda_{1}+1\right)\left(\gamma_{i, 3-k}-\gamma_{i, k}\right)\left(b_{i}^{*} s\right)^{\gamma_{i, k}}\right) \\
& \times\left(\sum_{l=1}^{2} \widehat{D}_{1-i, l}\left(s ; b_{1-i}^{*}\right)\left(\gamma_{i, 3-k}-\beta_{1-i, l}\right)\left(b_{i}^{*} s\right)^{\beta_{1-i, l}}+\frac{\lambda_{1-i}\left(1+(-1)^{i}\left(r+\lambda_{1-i}\right)\right) \gamma_{i, 3-k} L_{i} s}{r+\lambda_{1-i}}\right. \\
& \left.\quad-\frac{\lambda_{1-i}\left(1+(-1)^{i}\left(r+\lambda_{1-i}-\mu\right)\right)\left(\gamma_{i, 3-k}-1\right) K_{i} b_{i}^{*} s}{r+\lambda_{1-i}-\mu}\right)
\end{aligned}
$$


and

$$
\begin{aligned}
& \widehat{C}_{1-i, k}\left(s ; b_{i}^{*}, b_{1-i}^{*}\right)=\lambda_{i} /\left(\left(\lambda_{0} \lambda_{1}+1\right)\left(\gamma_{1-i, 3-k}-\gamma_{1-i, k}\right)\left(b_{1-i}^{*} s\right)^{\gamma_{1-i, k}}\right) \\
& \times\left(\sum_{l=1}^{2} \widehat{D}_{1-i, l}\left(s ; b_{1-i}^{*}\right)\left(\gamma_{1-i, 3-k}-\beta_{1-i, l}\right)\left(b_{i}^{*} s\right)^{\beta_{1-i, l}}+\frac{\left(\lambda_{0} \lambda_{1}-(-1)^{i}\left(r+\lambda_{1-i}\right)\right) \gamma_{1-i, 3-k} L_{i} s}{\lambda_{i}\left(r+\lambda_{1-i}\right)}\right. \\
& \left.\quad-\frac{\left(\lambda_{0} \lambda_{1}-(-1)^{i}\left(r+\lambda_{1-i}-\mu\right)\right)\left(\gamma_{1-i, 3-k}-1\right) K_{i} b_{i}^{*} s}{\lambda_{i}\left(r+\lambda_{1-i}-\mu\right)}\right)
\end{aligned}
$$

with $\widehat{D}_{1-i, k}\left(s ; b_{1-i}^{*}\right), k=1,2$, from (5.11). Then, by applying the condition of (5.6) to the functions in (5.12), we conclude that the values $b_{j}^{*}, j=0,1$, are determined from the system of arithmetic equations:

$$
\sum_{k=1}^{2}\left(\lambda_{j} \widehat{C}_{j, k}^{\prime}\left(s ; b_{i}, b_{1-i}\right) s^{\gamma_{j, k}}-(-1)^{j} \widehat{C}_{1-j, k}^{\prime}\left(s ; b_{i}, b_{1-i}\right) s^{\gamma_{1-j, k}}\right)=0
$$

with

$$
\widehat{C}_{j, k}^{\prime}\left(s ; b_{i}, b_{1-i}\right)=\left(1-\gamma_{j, k}\right) \widehat{C}_{j, k}\left(s ; b_{i}, b_{1-i}\right) / s
$$

where $\widehat{C}_{j, k}\left(s ; b_{i}^{*}, b_{1-i}^{*}\right)$, for $i, j=0,1$ and $k=1,2$, are given by the expressions in (5.13)-(5.14).

(ii) Let us now assume that $\lambda_{i}>\lambda_{1-i}=0$ holds, for any $i=0,1$ fixed. In this case, by using straightforward calculations from part (i) of Subsection 3.2, it can be shown that the candidate solution of the system in (5.3)-(5.9) takes the form:

$$
W_{1-i}\left(x, s ; b_{1-i}^{*}\right)=\widehat{C}_{1-i, 1}\left(s ; b_{1-i}^{*}\right) x^{\gamma_{0,1}}+\widehat{C}_{1-i, 2}\left(s ; b_{1-i}^{*}\right) x^{\gamma_{0,2}}
$$

for $0<b_{1-i}^{*} s<x \leq s$, with

$$
\widehat{C}_{1-i, k}\left(s ; b_{1-i}^{*}\right)=\gamma_{0,3-k}\left(L_{1-i} s-K_{1-i} b_{1-i}^{*} s\right) /\left(\left(\gamma_{0,3-k}-\gamma_{0, k}\right)\left(b_{1-i}^{*} s\right)^{\gamma_{0, k}}\right)
$$

for $k=1,2$, where the value $b_{1-i}^{*}$ is determined by the equation:

$$
b_{1-i}^{\gamma_{0,1}-\gamma_{0,2}}=\frac{\left(\gamma_{0,1}-1\right)\left(\gamma_{0,2}\left(L_{1-i}-K_{1-i} b_{1-i}\right)+K_{1-i} b_{1-i}\right)}{\left(\gamma_{0,2}-1\right)\left(\gamma_{0,1}\left(L_{1-i}-K_{1-i} b_{1-i}\right)+K_{1-i} b_{1-i}\right)}
$$

for $i=0,1$ (see [8]).

Substituting the expression for $W_{1-i}\left(x, s ; b_{1-i}^{*}\right)$ from (5.17) into the equation of (5.3) for $W_{i}(x, s)$ and applying the conditions of (5.4)-(5.6), we obtain that the candidate value function admits the representation:

$$
W_{i}\left(x, s ; b_{i}^{*}, b_{1-i}^{*}\right)=\sum_{k=1}^{2} \widehat{C}_{i, k}\left(s ; b_{i}^{*}, b_{1-i}^{*}\right) x^{\beta_{i, k}}+W_{1-i}\left(x, s ; b_{1-i}^{*}\right)
$$

for $0<b_{i}^{*} s<x \leq s$, with

$$
\widehat{C}_{i, k}\left(s ; b_{i}^{*}, b_{1-i}^{*}\right)=\frac{\partial_{x} W_{1-i}\left(b_{i}^{*} s, s ; b_{1-i}^{*}\right)-\beta_{i, 3-k}\left(W_{1-i}\left(b_{i}^{*} s, s ; b_{1-i}^{*}\right)-L_{i} s+K_{i} b_{i}^{*} s\right)}{\left(\beta_{i, 3-k}-\beta_{i, k}\right)\left(b_{i}^{*} s\right)^{\beta_{i, k}}}
$$


for $k=1,2$, and the value $b_{i}^{*}$ is determined by the arithmetic equation:

$$
\widehat{C}_{i, 1}^{\prime}\left(s ; b_{i}, b_{1-i}^{*}\right) s^{\gamma_{0,1}}+\widehat{C}_{i, 2}^{\prime}\left(s ; b_{i}, b_{1-i}^{*}\right) s^{\gamma_{0,2}}+\partial_{s} W_{1-i}\left(s, s ; b_{1-i}^{*}\right)=0
$$

where $W_{1-i}\left(s, s ; b_{1-i}^{*}\right)$ is given by $(5.17)$, for $i=0,1$.

(iii) Let us finally assume that $\lambda_{1-i}>\lambda_{i}=0$ holds, for any $i=0,1$ fixed. In this case, by using straightforward calculations from part (i) of Subsection 3.3, it can be shown that the candidate solution of the system in (5.3)-(5.9) takes the form:

$$
W_{i}\left(x, s ; b_{i}^{*}\right)=\widehat{C}_{i, 1}\left(s ; b_{i}^{*}\right) x^{\gamma_{0,1}}+\widehat{C}_{i, 2}\left(s ; b_{i}^{*}\right) x^{\gamma_{0,2}}
$$

for $0<b_{i}^{*} s<x \leq s$, with

$$
\widehat{C}_{i, k}\left(s ; b_{i}^{*}\right)=\left(\gamma_{0,3-k}\left(L_{i} s-K_{i} b_{i}^{*} s\right)+K_{i} b_{i}^{*} s\right) /\left(\left(\gamma_{0,3-k}-\gamma_{0, k}\right)\left(b_{i}^{*} s\right)^{\gamma_{0, k}}\right)
$$

for $k=1,2$, where the value $b_{i}^{*}$ is determined by the equation:

$$
b_{i}^{\gamma_{0,1}-\gamma_{0,2}}=\frac{\left(\gamma_{0,1}-1\right)\left(\gamma_{0,2}\left(L_{i}-K_{i} b_{i}\right)+K_{i} b_{i}\right)}{\left(\gamma_{0,2}-1\right)\left(\gamma_{0,1}\left(L_{i}-K_{i} b_{i}\right)+K_{i} b_{i}\right)}
$$

for $i=0,1$ (see $[8]$ ).

Substituting the function $W_{i}(x, s)=L_{i} s-K_{i} x$ into the equation of (5.3) for $W_{1-i}(x, s)$, by applying the conditions of (5.4)-(5.5), we obtain that the candidate value function $W_{1-i}\left(x, s ; b_{1-i}^{*}\right)$ admits the representation of (5.10), for $0<b_{1-i}^{*} s<x \leq b_{i}^{*} s$, with $\widehat{D}_{1-i, k}\left(s ; b_{1-i}^{*}\right), k=1,2$, given by (5.11), where we recall that $L_{1-i}^{\prime}=L_{1-i}-\lambda_{1-i} L_{i} /\left(r+\lambda_{1-i}\right)$ and $K_{1-i}^{\prime \prime}=K_{1-i}-$ $\lambda_{1-i} K_{i} /\left(r+\lambda_{1-i}-\mu\right)$, for $i=0,1$, fixed. Then, using the fact that the function $W_{1-i}(x, s)$ should be continuously differentiable at $b_{i}^{*} s$, we obtain the representation:

$$
W_{1-i}\left(x, s ; b_{i}^{*}, b_{1-i}^{*}\right)=\sum_{k=1}^{2} \widehat{C}_{1-i, k}\left(s ; b_{i}^{*}, b_{1-i}^{*}\right) x^{\beta_{1-i, k}}+W_{i}\left(x, s ; b_{i}^{*}\right)
$$

for $0<b_{i}^{*} s<x \leq s$, with

$$
\begin{aligned}
& \widehat{C}_{1-i, k}\left(s ; b_{i}^{*}, b_{1-i}^{*}\right) \\
& =\frac{\beta_{1-i, 3-k}\left(W_{1-i}\left(b_{i}^{*} s, s ; b_{1-i}^{*}, b_{i}^{*}\right)-L_{i} s+K_{i} b_{i}^{*} s\right)-\left(b_{i}^{*} s\right) \partial_{x} W_{1-i}\left(b_{i}^{*} s, s ; b_{1-i}^{*}, b_{i}^{*}\right)-K_{i} b_{i}^{*} s}{\left(\beta_{1-i, 3-k}-\beta_{1-i, k}\right)\left(b_{i}^{*} s\right)^{\beta_{1-i, k}}}
\end{aligned}
$$

for $k=1,2$, where $W_{1-i}\left(x, s ; b_{1-i}^{*}, b_{i}^{*}\right)$ is given by (5.10) with (5.11), and that the value $b_{1-i}^{*}$ is determined by the arithmetic equation:

$$
\widehat{C}_{1-i, 1}^{\prime}\left(s ; b_{i}^{*}, b_{1-i}\right) s^{\beta_{1-i, 1}}+\widehat{C}_{1-i, 2}^{\prime}\left(s ; b_{i}^{*}, b_{1-i}\right) s^{\beta_{1-i, 2}}+\partial_{s} W_{i}\left(s, s ; b_{i}^{*}\right)=0
$$

where $W_{i}\left(s, s ; b_{i}^{*}\right)$ is given by $(5.23)$, for $i=0,1$.

Summarising the facts proved above we formulate the following result which can be proved by means of the same arguments as Theorem 4.1 above. 
Theorem 5.1 Suppose that the assumptions of Theorem 4.1 hold. Then, the value function of the optimal stopping problem in (2.1) admits the representations:

$$
W_{i}^{*}(x, s)= \begin{cases}W_{i}\left(x, s ; b_{i}^{*}, b_{1-i}^{*}\right), & \text { if } b_{i}^{*} s<x \leq s \\ W_{i}\left(x, s ; b_{i}^{*}\right), & \text { if } b_{i}^{*} s<x \leq s \\ L_{i} s-K_{i} x, & \text { if } 0<x \leq b_{i}^{*} s\end{cases}
$$

and

$$
W_{1-i}^{*}(x, s)= \begin{cases}W_{1-i}\left(x, s ; b_{i}^{*}, b_{1-i}^{*}\right), & \text { if } \quad b_{i}^{*} s<x \leq s \\ W_{1-i}\left(x, s ; b_{1-i}^{*}\right), & \text { if } \quad b_{1-i}^{*} s<x \leq b_{i}^{*} s \\ L_{1-i} s-K_{1-i} x, & \text { if } 0<x \leq b_{1-i}^{*} s\end{cases}
$$

and the optimal stopping time has the form of (5.2), where the candidate value functions and linear boundaries are specified as follows:

(i): if $\lambda_{i}>0$, for every $i=0,1$, then $W_{j}\left(x, s ; b_{i}^{*}, b_{1-i}^{*}\right), j=0,1$, are given by (5.12), for $b_{i}^{*} s<x \leq s$, with $\widehat{C}_{j, k}\left(s ; b_{i}^{*}, b_{1-i}^{*}\right), j=0,1, k=1,2$, from (5.13)-(5.14), and $W_{1-i}\left(x, s ; b_{1-i}^{*}\right)$ is given by (5.10), for $b_{1-i}^{*} s<x \leq b_{i}^{*} s$, with $\widehat{D}_{1-i, k}\left(s ; b_{1-i}^{*}\right), k=1,2$, from (5.11), while $b_{j}^{*}$, $j=0,1$, are determined by the arithmetic equations in (5.15) with (5.16);

(ii): if $\lambda_{i}>\lambda_{1-i}=0$, for any $i=0,1$ fixed, then $W_{1-i}\left(x, s ; b_{1-i}^{*}\right)$ is given by (5.17), for $b_{1-i}^{*} s<x \leq s$, with $\widehat{C}_{1-i, k}\left(s ; b_{1-i}^{*}\right), k=1,2$, from (5.18), and $W_{i}\left(x, s ; b_{i}^{*}, b_{1-i}^{*}\right)$ is given by (5.20), for $b_{i}^{*} s<x \leq s$, with $\widehat{C}_{i, k}\left(s ; b_{i}^{*}, b_{1-i}^{*}\right), k=1,2$, from (5.21), while $b_{j}^{*}, j=0,1$, are determined by the arithmetic equations in (5.19) and (5.22);

(iii): if $\lambda_{1-i}>\lambda_{i}=0$, for any $i=0,1$ fixed, then $W_{i}\left(x, s ; b_{i}^{*}\right)$ is given by (5.23), for $b_{i}^{*} s<x \leq s$, with $\widehat{C}_{i, k}\left(s ; b_{i}^{*}\right), k=1,2$, from (5.24), $W_{1-i}\left(x, s ; b_{1-i}^{*}\right)$ is given by (5.10), for $b_{1-i}^{*} s<x \leq b_{i}^{*} s$, with $\widehat{D}_{1-i, k}\left(s ; b_{1-i}^{*}\right), k=1,2$, from (5.11), and $W_{1-i}\left(x, s ; b_{i}^{*}, b_{1-i}^{*}\right)$ is given by (5.26), for $b_{i}^{*} s<x \leq s$, with $\widehat{C}_{1-i, k}\left(s ; b_{i}^{*}, b_{1-i}^{*}\right), k=1,2$, from (5.27), while $b_{j}^{*}, j=0,1$, are determined by the arithmetic equations in (5.25) and (5.28).

Acknowledgments. The authors are grateful to the Associate Editor and two anonymous Referees for their useful suggestions which helped to essentially improve the presentation of the paper. Peter Kort and Maria Lavrutich acknowledge support from the Research Council of Norway through project nr. 268093.

\section{References}

[1] Adkins, R. and Paxson, D. (2016). Subsidies for renewable energy facilitiesunder uncertainty. The Manchester School 84 (222-250).

[2] Asmussen, S., Avram, F. and Pistorius, M. (2003). Russian and American put options under exponential phase-type Lévy models. Stochastic Processes and Applications 109 (79-111).

[3] Avram, F., Kyprianou, A. E. and Pistorius, M. (2004). Exit problems for spectrally negative Lévy processes and applications to (Canadized) Russian options. Annals of Applied Probability 14(1) (215-238).

[4] Boomsma, T. K. and Linnerud, K. (2015). Market and policy risk under different renewable electricity support schemes. European Journal of Operations Research 89 (435-448). 
[5] Boomsma, T. K., Meade, N. and Fleten, S.-E. (2012). Renewable energy investments under different support schemes: A real options approach. European Journal of Operations Research 220 (225-237).

[6] Baurdoux, E. J. and Kyprianou, A. E. (2009). The Shepp-Shiryaev stochastic game driven by a spectrally negative Lévy process. Theory of Probability and its Applications 53 (481-499).

[7] Baurdoux, E. J. and van Schaik, K. (2014). Predicting the time at which a Lévy process attains its ultimate supremum. Acta Applicandae Mathematicae 134(1) (21-44).

[8] Beibel, M. and Lerche, H. R. (1997). A new look at warrant pricing and related optimal stopping problems. Empirical Bayes, sequential analysis and related topics in statistics and probability (New Brunswick, NJ, 1995). Statististica Sinica 7 (93-108).

[9] Chronopoulos, M., Hagspiel, V. and Fleten, S-E. (2016). Stepwise green investment under policy uncertainty. The Energy Journal 37 (87-108).

[10] Dalang, R. C. and Hongler, M.-O. (2004). The right time to sell a stock whose price is driven by Markovian noise. Annals of Applied Probability 14 (2167-2201).

[11] Dalby, P. A. O., Gillerhaugen, G. R., Hagspiel, V., Leth-Olsen, T. and ThiJSSEN, J. J. J. (2018). Green investment under policy uncertainty and Bayesian learning. Energy 161 $(1262-1281)$.

[12] Dixit, A. K. and Pindyck, R. S. (1994). Investment under Uncertainty. Princeton University Press, New Jersey.

[13] Dubins, L., Shepp, L. A. and Shiryaev, A. N. (1993). Optimal stopping rules and maximal inequalities for Bessel processes. Theory of Probability and its Applications 38(2) (226-261).

[14] Elliott, R. J., Aggoun, L. and Moore, J. B. (1995). Hidden Markov Models: Estimation and Control. Springer, New York.

[15] Eryilmaz, D. and Homans, F. R. (2016). How does uncertainty in renewable energy policy affect decisions to invest in wind energy. The Electricity Journal 29 (64-71).

[16] Gapeev, P. V. (2007). Discounted optimal stopping for maxima of some jump-diffusion processes. Journal of Applied Probability 44 (713-731).

[17] Gapeev, P. V. and Rodosthenous, N. (2014). Optimal stopping problems in diffusion-type models with running maxima and drawdowns. Journal of Applied Probability 51(3) (799-817).

[18] Gapeev, P. V. and Rodosthenous, N. (2015). On the drawdowns and drawups in diffusion-type models with running maxima and minima. Journal of Mathematical Analysis and Applications 434(1) (413-431).

[19] Gapeev, P. V. and Rodosthenous, N. (2016). Perpetual American options in diffusion-type models with running maxima and drawdowns. Stochastic Processes and their Applications 126(7) (2038-2061).

[20] Gerber, H. U., Michaud, F. and Shiu, E. S. W. (1995). Pricing Russian options with the compound Poisson process. Insurance: Mathematics and Economics 17(1) (79-79).

[21] Glover, K., Hulley, H. and Peskir, G. (2013). Three-dimensional Brownian motion and the golden ratio rule. Annals of Applied Probability 23 (895-922).

[22] Graversen, S. E. and Peskir, G. (1998). Optimal stopping and maximal inequalities for geometric Brownian motion. Journal of Applied Probability 35(4) (856-872).

[23] Guo, X. (2001). An explicit solution to an optimal stopping problem with regime switching. Journal of Applied Probability 38 (464-481).

[24] Guo, X. and Shepp, L. A. (2001). Some optimal stopping problems with nontrivial boundaries for pricing exotic options. Journal of Applied Probability 38(3) (647-658).

[25] Guo, X. and Zervos, M. (2010). $\pi$ options. Stochastic Processes and Applications 120(7) (1033-1059). 
[26] Guo, X. and Zhang, Q. (2004). Closed-form solutions for perpetual American put options with regime switching. SIAM Journal on Applied Mathematics 64 (2034-2049).

[27] Hassett, K. A. and Metcalf, G. E. (1999). Investment with uncertain tax policy: does random tax policy discourage investment? The Economic Journal 109 (372-393).

[28] Jiang, Z. and Pistorius, M. R. (2008). On perpetual American put valuation and first-passage in a regime-switching model with jumps. Finance and Stochastics 12 (331-355).

[29] Jobert, A. and Rogers, L. C. G. (2006). Option pricing with Markov-modulated dynamics. SIAM Journal on Control and Optimization 44 (2063-2078).

[30] Kyprianou, A. E. and Отt, C. (2014). A capped optimal stopping problem for the maximum process. Acta Applicandae Mathematicae 129 (147-174).

[31] Liptser, R. S. and Shiryaev, A. N. (2001). Statistics of Random Processes I. (Second Edition, First Edition 1977) Springer, Berlin.

[32] Mordecki, E. and Moreira, W. (2001). Russian options for a difussion with negative jumps. Publicaciones Matemáticas del Uruguay 9 (37-51).

[33] Отт, C. (2013). Optimal stopping problems for the maximum process with upper and lower caps. Annals of Applied Probability 23 (2327-2356).

[34] Pedersen, J. L. (2000). Discounted optimal stopping problems for the maximum process. Journal of Applied Probability 37(4) (972-983).

[35] Peskir, G. (1998). Optimal stopping of the maximum process: The maximality principle. Annals of Probability 26(4) (1614-1640).

[36] Peskir, G. (2007). A change-of-variable formula with local time on surfaces. Séminaire de Probabilité $X L$. Lecture Notes in Mathematics 1899, Springer (69-96).

[37] Peskin, G. (2012). Optimal detection of a hidden target: The median rule. Stochastic Processes and their Applications 122 (2249-2263).

[38] Peskir, G. (2014). Quickest detection of a hidden target and extremal surfaces. Annals of Applied Probability 24(6) (2340-2370).

[39] Peskin, G. and Shiryaev, A. N. (2006). Optimal Stopping and Free-Boundary Problems. Birkhäuser, Basel.

[40] Rodosthenous, N. and Zervos, M. (2017). Watermark options. Finance and Stochastics 21(1) (157186).

[41] Revuz, D. and Yor, M. (1999). Continuous Martingales and Brownian Motion. Springer, Berlin.

[42] Ritzenhofer, I. and Spinler, S. (2016). Optimal design of feed-in-tariffs to stimulate renewable energy investments under regulatory uncertainty - a real options analysis. Energy Economics 53 (76-89).

[43] Shepp, L. A. and Shiryaev, A. N. (1993). The Russian option: reduced regret. Annals of Applied Probability 3(3) (631-640).

[44] Shepp, L. A. and Shiryaev, A. N. (1994). A new look at the pricing of Russian options. Theory Probability and Applications 39(1) (103-119).

[45] Shiryaev, A. N. (1999). Essentials of Stochastic Finance. World Scientific, Singapore. 